\title{
Alleviation of Cholesterol Gallstones by Lactobacillus Targeting the Gut Microbiota Depends on Global Farnesoid X Receptor-Mediated Bile Acid Metabolism
}

\section{Xinjian Wan ( $\sim$ slwanxinjian2020@126.com )}

Shanghai 6th Peoples Hospital Affiliated to Shanghai Jiaotong University School of Medicine https://orcid.org/0000-0002-4201-633X

\section{Xin Ye}

Department of Gastroenterology, Shanghai General Hospital,Shanghai Jiao Tong University School of Medicine

\section{Qian Zhuang}

Shanghai 6th Peoples Hospital Affiliated to Shanghai Jiaotong University School of Medicine

\section{Zhixia Dong}

Shanghai 6th Peoples Hospital Affiliated to Shanghai Jiaotong University School of Medicine

\section{Xiaoxin Wang}

Shanghai Key Laboratoryof Pancreatic Disease, Institute of Pancreatic Disease,Shanghai Jiao Tong University School of Medicine

\section{Shuang Shen}

Shanghai 6th Peoples Hospital Affiliated to Shanghai Jiaotong University School of Medicine

\section{Shan Wu}

Shanghai 6th Peoples Hospital Affiliated to Shanghai Jiaotong University School of Medicine

\section{Min Ning}

Shanghai 6th Peoples Hospital Affiliated to Shanghai Jiaotong University School of Medicine Jie Xia

Shanghai 6th Peoples Hospital Affiliated to Shanghai Jiaotong University School of Medicine Jingjing Wang

Shanghai Sixth Peoples Hospital Affiliated to Shanghai Jiaotong University School of Medicine

\section{Research}

Keywords: Cholesterol gallstone, Gut microbiota, Lactobacillus, FXR, FGF15, Bile acids

Posted Date: December 4th, 2020 
DOl: https://doi.org/10.21203/rs.3.rs-120683/v1

License: (c) (1) This work is licensed under a Creative Commons Attribution 4.0 International License. Read Full License 


\section{Abstract \\ Background}

Cholesterol gallstone (CGS) disease is characterized by an imbalance in bile acid (BA) metabolism and is closely associated with gut microbiota disorders. However, the role and mechanism by which probiotics targeting the gut microbiota attenuate CGS are still unknown. In this study, Lactobacillus reuteri CGMCC 17942 (LR) and L. plantarum CGMCC 14407 (LP) were individually administered to lithogenic diet (LD)fed mice at a dosage of $10^{9} \mathrm{CFU} /$ day for 8 weeks.

\section{Results}

Both Lactobacillus strains significantly reduced LD-induced gallstones, hepatic steatosis, and hyperlipidemia. These strains modulated serum BA profiles, with significantly decreased conjugated primary BA taurine- $\beta$-muricholic acid (T- $\beta$-MCA), an FXR antagonist. At the molecular level, LR and LP increased Farnesoid X Receptor (FXR) expression in the liver but not in the ileum, increased the levels of ileum and liver fibroblast growth factor 15 (FGF15) and liver FGFR4, small heterodimer partner (SHP), and subsequently reduced cholesterol 7a-hydroxylase (CYP7A1) and cytochrome P450 family 7 subfamily B polypeptide 1 (CYP7B1) to inhibit BA synthesis in the liver. At the same time, the two strains enhanced BA transport by increasing the levels of multidrug-resistance-associated protein homologs (MRP) 3/4, multidrug-resistance-associated protein homologs (MRP) 3/4, hepatic multidrug resistance protein (MDR2) and bile salt export pump (BSEP) mRNA in the liver. In addition, both LR and LP reduced LDassociated gut microbiota dysbiosis. LR increased the relative abundance of Muribaculaceae, while LP increased that of Akkermansia. The changed gut microbiota was significantly negatively correlated with the grade and incidence of gallstones, hyperlipidemia, the level of T- $\beta$-MCA in serum, or the gene expression levels of Fxr in liver. Furthermore, the protective effects of the two strains were abolished by a global but not intestinal-specific FXR antagonist.

\section{Conclusions}

Taken together, our results suggested that Lactobacillus might relieve gallstones through FXR-dependent regulation of BA synthesis and transport.

\section{Background}

The incidence rate of cholesterol gallstone (CGS) formation is increasing with the popularity of Western food[1, 2]. CGSs are caused by cholesterol precipitation in the gallbladder or biliary tract, which can lead to more serious diseases, such as cholecystitis, cholangitis, pancreatitis, gallbladder carcinoma and colon cancers $[3,4]$. There is currently no specific medicine available, and surgical treatment has a high 
risk of recurrence[2]. Therefore, it is necessary to find effective drugs or methods to prevent or treat gallstones.

Gut microbiota dysbiosis and bacterial community assembly were associated with cholesterol gallstones in a large-scale study[5-7]. It has been reported that the proportion of Lactobacillus is greatly reduced in CGS mouse models[8]; however, whether Lactobacillus supplementation can reduce gallstones has not been studied. Yoshika Takeda and colleagues showed that Clostridium butyricum supplementation can alleviate the formation of gallstones in experimental CGS[9], but they did not state the mechanism by which this strain alleviated gallstones.

Bile acids (BAs) are biosynthesized from cholesterol by the liver, conjugated in hepatocytes, secreted into the small intestine through the gallbladder, hydrolyzed and dehydroxylated by the gut microbiota, and reabsorbed into the liver in the intestine through the portal system[10]. The change in the content or composition of BAs leads to the precipitation of cholesterol, which is considered to be an important cause of gallstones[11]. The gut microbiota-mediated transformation of the BA pool regulates the activation of nuclear receptor Farnesoid X Receptor (FXR) pathway signaling[10, 12]. FXR, as a member of the steroid/thyroid hormone receptor family of ligand-activated transcription factors, is an important BA receptor governing bile, glucose and lipid metabolism[10,13]. The current research indicates that FXR protects against CGS formation by regulating the hydrophobicity of BAs to promote the excretion of cholesterol[14, 15], inhibit the biosynthesis of BAs and cholesterol and reduce cholesterol crystal precipitation[10, 13]. Antonio Moschetta and colleagues showed that FXR activation promotes the transcription of small heterodimer partner (SHP) and fibroblast growth factor 15 (FGF15) genes to regulate $B A$ anabolism[16, 17]. Accumulating evidence in other disease models (not CGS) indicates that oral supplementation with probiotics, such as Lactobacillus rhamnosus GG, L. casei YRL577, and VSL\#3, can suppress hepatic BA synthesis and enhance BA deconjugation or excretion through the FXR-FGF15 signaling pathway in mice[12,16,18,19]. However, whether the effects of probiotics on CGS depend on the FXR-FGF15 axis is unknown.

In this study, our data showed that supplementation with L. reuteri CGMCC 17942 (LR) and L. plantarum CGMCC 14407 (LP) effectively prevented CGS formation. These strains ameliorated gut microbiota dysbiosis by increasing the relative abundance of Muribaculaceae or Akkermansia. They maintained the homeostasis of BA metabolism, primarily by reducing T- $\beta$-MCA to increase FXR expression and to reduce BA synthesis and transport in the liver. The effects of the two Lactobacillus strains on CGS depended on FXR activation.

\section{Results}

\section{LR and LP treatments reduced LD-induced gallstones and metabolic disorders in mice}


We hypothesized that the probiotics LR and LP may be protective against LD-induced gallstones. Thus, mice were fed LD for 8 weeks, and LR and LP were supplemented once a day from the beginning of the experiment. The gallbladders of mice fed a ND were filled with clear bile and without any cholesterol crystals. Round gallstones or stratified crystals and a small amount of sticky bile occupied the gallbladders of mice fed the LD. The incidence of gallstones reached $100 \%$. Only a few leaflet crystals or some cholesterol particles that were invisible to the naked eye and viscous bile were observed in the gallbladders of mice with LR and LP treatments, and the incidence of gallstones was markedly reduced (Fig. 1A, B). We used grading standards developed by Takashi Akiyoshi and his colleagues to evaluate the gallstones[20]. We found that the grade of the experimental gallstones in mice fed the LD was significantly higher than that of mice fed the ND and was decreased noticeably with LR and LP treatment (Fig. 1C). Compared to ND mice, LD mice showed severe changes in the gallbladder. The gallbladder volume and gallbladder wall thickness were increased significantly in mice fed the LD, and these changes in the gallbladder were markedly improved by LR and LP treatment (Fig. 1D, E), suggesting that LR and LP treatment can reduce the incidence of gallbladder stones and gallbladder wall hypertrophy.

Furthermore, several lines of evidence suggest that metabolic syndrome (MS) is associated with the morbidity of gallstones[21, 22]. We monitored the weights of mice once a week and found that the weights of LD-fed mice were significantly increased compared with those of ND-fed mice. The increase in body weight was reversed by LR and LP treatment, and LP treatment tended to show better results (Fig. 1F). We next calculated the ratio of liver weight to body weight in mice. The liver weight was increased, and the ratio of liver weight to body weight was significantly increased in the LD group compared with the ND group, but were significantly decreased by LR treatment. There was no significant decrease in liver weight after oral LP treatment, likely because the significant weight loss of mice in the LP group led to an increase in the ratio of liver weight to body weight (Fig. 1G). It was reported that insulin resistance is closely related to the onset of gallstones[22, 23]. Therefore, we further examined the serum glucose and insulin functions of mice by the oral glucose tolerance test (OGTT). We found that the hyperglycemia of LD-fed mice was significantly improved by LR and LP treatment. Furthermore, glucose tolerance was severely impaired in mice in the LD group compared to those in the ND group and was effectively improved by LR and LP treatment (Supplemental Fig. 1A, B), suggesting that treatment with LR and LP could reduce insulin resistance and that the therapeutic effects of the two probiotics are comparable in LD-fed mice.

Next, we examined whether LR and LP improved hepatic steatosis and protected against metabolite abnormalities in serum. In LD-fed mice, we found that there were many empty bubbles in the liver because of the accumulation of lipid droplets in the liver. LR and LP treatments led to a marked reduction in liver histological damage. Oil red staining was used to further confirm the change in lipids in the liver parenchyma. Compared to the livers of ND-fed mice, the livers of LD-fed mice showed many fat droplets stained with oil red, and the number of fat droplets was significantly decreased by LR and LP treatment (Fig. 1H). These data suggested that the oral administration of LR and LP can ameliorate hepatic steatosis, which is associated with cholesterol gallstones[24]. Serum alanine aminotransferase (ALT), aspartate aminotransferase (AST) and alkaline phosphatase (ALP) were similarly reduced in the LR and 
LP treated groups (Fig. 1I-J), suggesting that hepatic injury was ameliorated. Serum total cholesterol and triglycerides were also reduced by LR and LP treatment (Fig. 1K, L). Both HDL and LDL were increased in LD-fed mice compared with ND-fed mice, and this effect was reversed by LR treatment. Under LP treatment, HDL was further increased, while LDL was reduced (Fig. 1M).

\section{LR and LP treatments changed the bile acid composition in serum and activated FXR-FGF-15 signaling.}

Serum bile acids (BAs), as important signaling molecules, participate in the regulation of BA metabolism and the activation of several kinds of bile acid receptors[25]. Most BAs come from reabsorption in the intestinal epithelium after being processed by gut microbes and then entering the enterohepatic circulation[26, 27]. Therefore, we measured total bile acid (TBA) and the composition of BAs in serum to examine differences induced by LR and LP treatment. We found that serum TBA was markedly increased in LD-fed mice compared with ND-fed mice and was similarly decreased by oral LR treatment, but there was no significant difference in the reduction in TBA induced by LP treatment. Furthermore, the composition of BAs in the serum of LD-fed mice was significantly different from that in ND-fed mice, and many kinds of BAs that can activate FXR were significantly reduced, such as taurochenodeoxycholic acid (TCDCA), the most potent endogenous FXR agonist with an EC50 of $17 \mu \mathrm{M}[28]$, and chenodeoxycholic acid (CDCA). This effect was reversed by LR and LP treatments. Then, we further found that tauro-amuricholic acid (T-a-MCA) and tauro- $\beta$-muricholic acid (T- $\beta-M C A)$ were significantly upregulated in the LD group compared with the ND group, and the levels of both were reversed with the LR and LP treatments. The proportion of taurine-conjugated bile acids, including T- $\alpha-M C A$ and T- $\beta-M C A$, was significantly upregulated in LD-fed mice compared with ND-fed mice and downregulated with LR and LP treatments (Fig. 2A-C). T- $\alpha-M C A(I C 50=28 \mu \mathrm{M})$ and T-B-MCA $(I C 50=40 \mu \mathrm{M})$ are both known as efficient natural antagonists of FXR[28]. They can competitively inhibit the activation of FXR by other BAs[16]. The primary BAs cholic acid (CA) and CDCA are synthesized by the classic pathway and alternative pathway, respectively, and they are then translated into the secondary BAs deoxycholic acid (DCA) and lithocholic acid (LCA)[29]. The results showed that the serum CA, CDCA and DCA levels were decreased significantly in the LD-fed mice compared with the ND-fed mice, and the CA and DCA were decreased further with LR and LP treatments. Contrary to this result, CDCA and LCA were upregulated with LR and LP treatments, although there was no statistically significant difference in the upregulation by LP treatment (Fig. 4D). These results suggest that serum CA did not increase with dietary CA with the administration of the LD. However, the accumulation of high concentrations of CA in the intestine may activate intestinal FXR, which inhibits BA synthesis in the liver. Under these conditions, the alternative pathway slightly dominates the synthesis of BAs after LR and LP treatments. It is well known that the gut microbiota is involved in bile acid metabolism[27, 30]. Thus, we calculated the ratio of the sum of primary BAs to the sum of secondary BAs and found that the ratio was decreased, although the difference was not statistically significant (Supplemental Fig. 2A). The results indicated that the secondary BAs were increased by the LR and LP treatments.

It has been reported that the downregulation of T-a-MCA and T- $\beta-M C A$ can reverse the inhibition of FXR and activate the FXR-FGF15/SHP pathway[16, 28]. In the current study, we examined the mRNA 
expression of ileum Fgf15, Fxr, and Shp and the protein levels of FGF15 and FXR. We found that FGF15 expression in the ileum was markedly decreased in LD-fed mice compared with ND-fed mice, as detected by immunofluorescence, and increased significantly with LR and LP treatments (Fig. 2E). We found that the mRNA levels of ileum Shp and Fgf15 were both upregulated in LD-fed mice compared with ND-fed mice, and there was a more marked increase in Shp and Fgf15 with the LR and LP treatments. The protein level of FGF15 was consistent with the mRNA level. The mRNA level of ileum FXR in LD-fed mice was not significantly different from that in ND-fed mice, but it was significantly decreased with LR and LP treatments; however, the changes were not significant at the protein level (Fig. 2F, G). A high dose of CA in the ileum could have led to ileum FXR activation in LD-fed mice[28]. However, the high contents of T-aMCA and T- $\beta$-MCA in the LD group inhibited the activity of FXR and had no effect on the mRNA level of FXR. The inhibition relieved by LR and LP treatment may lead to FXR overactivation, causing a decrease in the adaptability of FXR mRNA levels in the ileum. Next, we examined the mRNA level of ileal bile acid transporter (IBAT), also known as ABST, which plays an important role in reabsorbing BAs from the intestine to the enterohepatic circulation[31]. We found that the expression of IBAT was restored by LR and LP treatments, suggesting that the reabsorption of BAs was improved.

Furthermore, we found that the administration of the LD did not change the mRNA and protein levels of liver FXR; however, the protein level of liver FXR was increased significantly with LR and LP treatments. We found that the mRNA level of liver Shp and protein level of liver FGF15 were increased by LR and LP. Then, we examined the mRNA and protein levels of liver FGFR4, an important receptor in the FXR signaling pathway, and found that the mRNA and protein expression of FGFR4 was markedly reduced in LD-fed mice compared with ND-fed mice and that LR and LP treatments could reverse it, indicating that LR and LP treatments repaired the FXR-FGFR4 signaling pathway. The obstruction of the transport of bile acids to the gall bladder is a risk for the formation of gallstones[32]. The multidrug-resistance-associated protein homologs (MRPs) in the basolateral membrane of hepatocytes are responsible for excreting BAs[33]. We found that the mRNA expression of MRP4, a transporter that mediates the coeffelux of conjugated BAs[34], was significantly decreased by the LD, and the LR and LP treatments markedly upregulated it. The mRNA levels of MRP3 and hepatic multidrug resistance protein (MDR2) were significantly increased in LD-fed mice, and there was a more marked increase with LR or LP treatment, indicating that LR and LP intervention improve biliary phospholipid secretion[32]. However, the expression of MRP2 was not significantly changed in LD-fed mice regardless of LR or LP treatment (Supplemental Fig. 2B). The expression of the bile salt export pump (BSEP) was detected and found to be decreased in LD-fed mice compared with ND-fed mice; LR and LP treatments reversed this decrease. (Fig. 2H, I).

To study the downstream signaling pathway of FXR, we assessed the expression of cholesterol 7ahydroxylase (CYP7A1), sterol 12a-hydroxylase (CYP8B1), Sterol 27-hydroxylase (CYP27A1) and oxysterol 7a-hydroxylase (CYP7B1), which are important catalytic enzymes for the synthesis of BAs from cholesterol[35, 36]. Then, we found that CYP7A1, a rate-limiting enzyme of the classical pathway of BA synthesis[37], was increased in LD-fed mice compared with ND-fed mice. After the LR and LP treatments, the level of CYP7A1 was reduced markedly, suggesting that LR and LP treatments can inhibit BA synthesis via the classical pathway. The protein level of CYP8B1, another important enzyme in the 
classical pathway, showed no significant change with the LD, LR and LP interventions. The mRNA level of CYP8B1 was decreased by the LD, and the LR and LP treatments had no effect on it. Furthermore, as a rate-limiting enzyme in the alternative pathway, CYP27A1 was examined. The mRNA and protein levels of CYP27A1 were upregulated in LD-fed mice compared with ND-fed mice, and there was a more marked increase with LR and LP treatments, indicating that the alternative pathway was not inhibited completely. The changes in CYP7B1 were consistent with the changes in CYP7A1 (Fig. 2J, K).

To further study the secretion of cholesterol in the liver, we assessed the expression of hepatic cholesterol excretion genes ATP-binding cassette subfamily $G$ member 5 and 8 (ABCG5/8)[38]. We first found that the mRNA level of ABCG5/8 was increased in LD-fed mice compared with ND-fed mice, and it could be reversed by LR treatment but not LP treatment (Supplemental Fig. $2 \mathrm{C}$ ), suggesting that the LR and LP treatments exerted different effects on cholesterol transport. Next, we found that the mRNA and protein levels of sterol-regulatory element binding protein 2 (SREBP2) and SREBP cleavage-activating protein (SCAP) were markedly upregulated in LD-fed mice compared with ND-fed mice and that the LR and LP treatments significantly reversed these effects (Supplemental Fig. 2D-E). However, we did not observe any significant changes in 3-Hydroxy-3-Methylglutaryl-CoA Reductase (HMGCR), a cholesterol synthesis ratelimiting enzyme, with the LR and LP treatments (Supplemental Fig. 2F),

Collectively, these data demonstrate that BA synthesis was inhibited through the FXR-FGF15/SHP-FGFR4 signaling pathway in the liver by LR and LP treatments and that the alternative pathway is more advantageous than the classical pathway. The excretion of bile acid into the gallbladder was promoted. Consistently, cholesterol synthesis was inhibited by downregulating the expression of SCAP and SREBP2[39], and cholesterol efflux was suppressed.

\section{LR and LP treatments changed the CGS-associated gut microbiota composition in LD-fed mice}

To further validate our hypothesis that LR and LP alleviated CGS by rebuilding the intestinal flora, we collected feces from mice for analysis of the V3-V4 region of the 16S rRNA genes with an Illumina platform. Principal coordinate analysis (PCOA), a visual method used to study the differences between bacterial communities, indicated that the operational taxonomic units (OTUs) of the LD group were significantly different from those of the ND group, and orally administered LR and LP made the microbial composition of the LD-fed mice more similar to that of the ND-fed mice at the OTU level (Fig. 3A). Then, we used analysis of similarities (ANOSIM) to compare the similarities of the bacterial communities at the phylum level between samples based on Bray-Curtis analysis. The distance between groups was larger than all the intragroup distance, which indicated that the differences between groups were significantly greater than those within the groups (ANOSIM, P < 0.001) (Fig. 3B). At the phylum level, after 8 weeks of feeding the LD, the relative abundance of Firmicutes, the most common phylum in most people[40], was increased from 5198 to 14172 compared with that of the ND group and significantly decreased to 10706 and 10307 with oral LR and LP administration, respectively. In contrast, Bacteroidetes and Verrucomicrobia decreased from 12935 and 2128 to 6383 and 249, respectively, and the effects of the LR 
and LP treatments were the opposite (Fig. 3C). We took a closer look at the Firmicutes/Bacteroidetes $(\mathrm{F} / \mathrm{B})$ ratio, which is always associated with obesity[41]. We found that the $\mathrm{F} / \mathrm{B}$ ratio was significantly increased in LD-fed mice compared with ND-fed mice and that this differences was reversed by LR treatment. Contrary to LR treatment, LP treatment did not induce a decrease in the F/B ratio (Fig. 3D).

At the genus level, the Venn diagrams indicated that the proportion of approximately $48.61 \%$ of the total species was identical among the four groups (Supplemental Fig. 3A), revealing that the core microbiota remained stable. At the family level, community bar plot analysis showed that the percentages of community abundances of Muribaculaceae and Akkermansiaceae were both downregulated significantly in LD-fed mice compared with ND-fed mice. The abundance of Muribaculaceae slightly recovered with LR treatment, and that of Akkermansiaceae was greatly upregulated with LP treatment. Muribaculaceae occupies a major proportion of the feces of healthy mice and plays a critical role in improving metabolic disorders[42, 43]. It is reasonable to speculate that metabolic disorders in LD-fed mice are associated with Muribaculaceae and alleviated with LR treatment. In recent years, a number of studies have revealed that Akkermansiaceae has an important function in improving metabolic syndrome and reducing intestinal inflammation[44, 45], and our results showed that the reductions in Akkermansiaceae in LD-fed mice were reversed by LP treatment. Furthermore, we observed a significantly increased abundance of Erysipelotrichaceae in the LD group compared to the ND group, which was reversed by LR and LP treatments (Fig. 3E, F). The reduction in Erysipelotrichaceae is beneficial to lipid metabolism[46]. As the Kruskal-Wallis $\mathrm{H}$ test bar plot in Fig. 3F shows, Lactobacillaceae, which was significantly decreased in LDfed mice, was upregulated by LR and LP interventions. We further identified the difference in Lactobacillus at the species level. The Kruskal-Wallis $\mathrm{H}$ test bar plot of Lactobacillus showed that $L$. reuteri was upregulated significantly with LR treatment, and L. plantarum subsp. plantarum was upregulated significantly with LP treatment (Supplemental Fig. 3B). We further examined the species (top 50 abundances) in the feces of mice and found that the abundance of several species was similarly changed in the LR and LP treatments; however, most species had their own characteristics in the LR and LP groups (Fig. 3G).

These results indicate that LR and LP treatments manipulated the gut microbiota composition at the phylum, genus, family and species levels in LD-fed mice, leading increasing the number of metabolismpromoting flora and inducing a shift in the microbiota abundance patterns from LD-induced metabolic disorders to healthy conditions in different ways.

\section{Correlation between gut microbiota and host CGS-related parameters}

To further investigate the association of gut microbiota modulation by LR/LP and CGS, Spearman's correlation analysis was performed between the top 50 most abundant species and families that were changed by the two strains and several CGS-related parameters in all the groups of mice. At the species level, almost a quarter of these OTUs were significantly correlated with incidence and the grade of CGS, and one in ten of these OTUs was negatively correlated with them (Fig. 4A). At the family level, only 7 
OTUs and 9 OTUs were correlated with incidence, and the grade of CGS and 10 OTUs and 8 OTUs were negatively correlated with them (Supplemental Fig. 4A). Next, we found that the top 50 most abundant microbes at the species and families were significantly correlated with TBA and the composition of BAs (Fig. 4B, Supplemental Fig. 4B). Furthermore, we used Spearman's correlation analysis to investigate the relationship between several serum biomarkers and gut microbiota. The results showed that 6 of the 50 OTUs at the species level that were negatively correlated with serum ALP, AST, and ALT were enriched, including bacteria belonging to Akkermansia, Muribaculaceae, Muribaculum, and Turicibacter, and 12 of the 50 OTUs at the family level that were negatively correlated with serum ALP, AST, and ALT, namely, Muribaculaceae, Bacteroidales, Akkermansiaceae, Moraxellaceae, Aerococcaceae, Lactobacillaceae, Defluviitaleaceae, Clostrediates_vadinBB60_group, Mollicutes_RF39, Saccharimonadaceae, Caldicoprobacteraceae, and Anaeroplasmataceae, suggesting that these bacteria repair liver injury. We found that some gut microbes were significantly positively correlated with serum TG, TC, HDL, LDL, and Glu, such as Alloprevotella, Dubosiella, Rikkenella, Roseburia, Erysipelatoclostridium, at the species level, and Erysipelotrichaceae, Family_XIII, Atopobiaceae and so on at the family level were suggested to aggravate metabolic disorders (Fig. 4C, Supplemental Fig. 4C). Finally, we found that at both the species and family levels, the correlations between gut microbes and the mRNA levels of Cyp7a1, Cyp7b1, Cyp8b1 and ileum Fxr were similar and contrary to the mRNA levels of Cyp27a1, Mdr2, ileum Fgf15, ileum Shp, liver Shp, and liver Mrp3 (Fig. 4D, Supplemental Fig. 4D). Taken together, these data demonstrate that the CGS phenotypes and the composition of serum BAs, TBA, TC, TG, Glu, AST, ALT, ALP, HDL and LDL were significantly correlated with gut microbiota and that LR and LP treatments exerted protective effects on maintaining the balance of metabolism by regulating the gut microbiota.

\section{Inhibition of FXR activation attenuated the protective effects of LR and LP in LD-fed mice}

The data presented above indicate a critical involvement of FXR in LR- and LP-mediated prevention of gallstones in LD-fed mice. We hypothesized that the beneficial effects of LR and LP treatments could be abolished by the inhibition of FXR activation. To test this hypothesis, mice administered the LD, ND or LR and LP treatments for 8 weeks were gavaged with (Z)-guggulsterone (Z-Gu), a global FXR inhibitor, or glycine- $\beta$-muricholic acid (Gly-MCA), an intestine-specific FXR inhibitor[12]. Compared to the round gallstones and sticky bile observed in the gallbladders of LD-fed mice, we found that only a few leaflet crystals or some cholesterol particles in the gallbladders of LR- and LP-treated mice, and the incidence of gallstones and the grade of gallstones could be reduced by LR and LP treatments. However, Z-Gu almost completely reversed these protective effects, and granular stones filled the gallbladder. Gly-MCA also weakened the protective effects, and several leaflet crystals were suspended in bile. Under the Z-Gu intervention, the quantity and quality of gallstones were higher, and the incidence of gallstones was higher than that of the Gly-MCA intervention (Fig. 5A-C), suggesting that global FXR plays a more important role than intestinal FXR. Then, we calculated the ratio of liver weight to body weight in mice and found that inhibiting FXR by Z-Gu and Gly-MCA treatment could significantly reverse the LR-induced reduction in the liver weight to body weight ratio, but there were no significant changes in LP-treated mice (Fig. 5D, E). The LR- and LP-induced reductions in the volume of the gallbladder and the thickness of the 
gallbladder wall were markedly reversed by the Z-Gu and Gly-MCA interventions, and the results showed that inhibiting global FXR, but not intestine-specific FXR, could make the gallbladder wall thicker than LD feeding alone (Fig. 5F, G). A likely reason is that inhibiting global FXR instead of inhibiting intestinespecific FXR may lead to gallbladder wall thickening.

H\&E staining and Oil red O staining were performed to examine hepatic steatosis. Consistently, we found that Z-Gu abolished the protective effects of the LR and LP treatments and that Gly-MCA slightly blunted these effects (Fig. 5H), suggesting that global FXR was necessary for LR or LP to improve liver fat metabolism and that intestine-specific FXR plays only synergistic roles. Serum ALT, AST and ALP were analyzed, and we found that both LR and LP intervention reversed the decreases in ALT, AST and ALP caused by LR or LP treatment and was more effective (Fig. 5I, J), indicating that inhibiting global FXR may almost completely abolish the protective effects of LR and LP in LD-fed mice. We found that LR and LP treatment effectively reduced serum TC and TG, and the intestine-specific FXR inhibitor Gly-MCA significantly reversed these effects. However, the results showed that inhibition of serum TC and TG by LR and LP was not affected by global FXR inhibitors (Fig. 8K, L). Compared with LP treatment, treatment with Z-Gu and Gly-MCA reduced serum HDL, although the changes were nonsignificant (Fig. 8N). Conversely, the serum LDL level was upregulated with Z-Gu and Gly-MCA interventions (Fig. 80). Consistent with previous research results[47], the Z-Gu and Gly-MCA interventions abolished the protective effect of LR and LP treatments on serum glucose (Supplemental Fig. 5A). We further found that while the serum TBA was reduced by the LR and LP treatments, the Z-Gu completely reversed this effect, and Gly-MCA did not affect it (Fig. 8N), indicating that the LR and LP treatment-induced decrease

in TBA in LD-fed mice occurred through global FXR pathway. Taken together, these data demonstrate that the LR and LP treatments ameliorate experimental gallstones at least partly in an FXR-dependent manner.

\section{Discussion}

Although CGS patients and mice had a low abundance of Lactobacillus[8, 48], whether Lactobacillus can mitigate CGS and its mechanism remain unclear. In this study, we found that 1 ) one $L$. reuteri strain and one $L$. plantarum strain significantly alleviated CGS and its related fat deposition in the liver and serum, which depended on global FXR but not intestine-specific FXR; 2) the two Lactobacillus strains reduced the serum total BA pool, especially T- $\beta$-MCA, an FXR antagonist, and then decreased BA synthesis and transport in the liver by increasing liver FXR, FGF15 and FGFR4 and ileum FGF15; and 3) the two strains slightly reversed LD-induced CGS-associated gut microbiota dysbiosis, including the increases in Muribaculaceae by $L$. reuteri and Akkermansia by L. plantarum. All these results suggested that Lactobacillus prevented CGS through the FXR-FGF15-FGFR4 pathway.

Our study showed that one of the most important mechanisms by which the LR and LP treatments reduced the incidence of gallstones is the activation of the FXR-FGF15-FGFR4 signaling pathway. We found that T- $\beta$-MCA was markedly increased and that FXR protein levels were inhibited, although there were no statistically significant differences in LD-fed mice. Jingli Cai and colleagues showed that the mRNA level of FXR in patients with cholelithiasis was lower than that in control individuals[14], 
suggesting that the inhibition of FXR signaling is very likely to promote the onset of CGS. Loss of FXR signaling is one of the major risk factors for CGS formation because it destroys the FXR-driven negative feedback regulation of BA synthesis and leads to an imbalance in the BA pool[17]. We found that the LR and LP treatments downregulated T- $\beta$-MCA to activate FXR signaling, leading to several metabolic effects that could reduce the risk of gallstone formation. The increased ileal expression of FGF15 and hepatic expression of FGFR4 in LR/LP-treated mice was caused by the activation of FXR, which could inhibit the expression of the rate-limiting enzyme CYP7A1 to reduce BA synthesis. Furthermore, we found that the levels of hepatic MRP4, MDR2, and BSEP and ileal IBAT were upregulated by the LR and LP treatments, indicating that BA drainage to the gallbladder and intestinal reabsorption both increased to prevent the precipitation of cholesterol from bile.

It should be noted that the protective effects of the two strains on CGS were almost completely blocked by a global FXR inhibitor but not intestine-specific FXR. This phenomenon suggested that intestinal FXR did not protect the host from CGS after Lactobacilus administration, while global FXR, especially liver FXR, did. Liver FXR expression might be upregulated by Lactobacillus by downregulating T- $\beta$-MCA in serum. It was reported that Lactobacillus casei YRL577 alleviated nonalcoholic fatty liver disease in mice by increasing the intestinal FXR-FGF15 pathway[49], and Yunhuan Liu and colleagues found that FXR in the liver and intestine activated by Lactobacillus rhamnosus GG had a synergistic effect to reduce cholestasis[12]. However, Insook Ki and colleagues showed the differential regulation of BA homeostasis by FXR in the liver and intestine and used tissue-specific Fxr-null mice to suggest the more effective regulation of BA metabolism-related enzymes by liver FXR[50]. This conclusion also supports our results that liver FXR signaling plays a pivotal role in CGS prevention.

Previous studies have proven that the gut microbiota and BA metabolism are mutually regulated by each other. The amount of secondary BAs was dependent on primary BA deconjugation and subsequent conversion by the gut microbiota[27]. The conversion of conjugated BAs converted to deconjugated BAs is dependent on gut microbiota catalyzing the reaction[51]. Furthermore, the conjugated BAs will inhibit the growth of gut microbiota. In this study, we found that the intestinal flora was disrupted and that several metabolic disorders were present in LD-fed mice. LR and LP improved these disorders in different ways. LR treatment mainly played the role of increasing the diversity of the intestinal flora and restoring the proportion of Muribaculaceae. It is worth noting that LP treatment did not affect the diversity of the intestinal flora but greatly increased the proportion of Akkermansia to improve metabolic disorders. This phenomenon is consistent with our previous research results[52]. This is likely the reason by which LP produces some metabolites that promote the growth of Akkermansia. Maria and colleagues showed that Akkermansia abundance is associated with a healthier metabolic status[45]. Clara and colleagues demonstrated that supplementation with Akkermansia can improve obesity-related disorders and liver injury while maintaining the gut microbiome structure balance[53]. This phenomenon has revealed that some probiotics might show their effects by modulating the inherent host gut microbiota. Even though LR and LP are Lactobacillus strains and both have protective effects against CGS formation, the effects of the two on the intestinal flora were quite different, suggesting the functional redundancy of the gut microbiota. 


\section{Conclusions}

In summary, we demonstrated that gut microbiota-targeted LR and LP treatments reduced the concentration of T- $\beta$-MCA and promoted FXR activation to reduce CGS formation. The reduction in T- $\beta$ MCA relieved the competitive inhibition of FXR and upregulated the expression of ileal and hepatic FGF15 and hepatic FGFR4 to inhibit BA synthesis and promote the excretion of BAs to the gallbladder. Although additional research is needed before translation to patients, supplementation with LR or LP could be a promising therapeutic strategy for treating CGS or preventing the recurrence of CGS.

\section{Materials And Methods}

Mouse strains and treatments

Eight- to ten-week-old male C57BL/6J mice weighing 20-22 g were purchased from Shanghai SLAC Laboratory Animal Co Ltd. (Shanghai, China). All mice were maintained under 12-hour light/dark cycles at $22^{\circ} \mathrm{C}$ with unlimited access to a standard rodent diet and water and were housed under specific pathogen-free (SPF) conditions (free of known bacteria, including Helicobacterspp., viral and parasitic pathogens). Mice were allowed to acclimate for at least one week before experiments. All animal experiments were conducted under the"3R"principle (reduction, replacement and refinement) and were approved by the Animal Ethics Committee of Shanghai Jiao Tong University School of Medicine (SYXK 2013-0050, Shanghai, China). A lithogenic diet-induced mouse model of CGS was used in this study. Mice were fed a standard chow diet (normal diet, ND, containing $0.02 \%$ cholesterol) or a lithogenic diet (LD, containing $15 \%$ butter fat, $1.25 \%$ cholesterol and $0.5 \%$ cholic acid) for 8 weeks ( $n=12$ mice/group)[54]. To determine the effect of Lactobacillus reuteri CGMCC 17942 (LR) and Lactobacillus plantarum CGMCC 14407 (LP), the mice fed an LD received $200 \mu \mathrm{L}$ of bacterial suspension of either LP or LR at a dosage of $10^{9}$ colony-forming units (CFU)/day for 8 weeks. The ND group and LD group received an equal volume of normal saline, which served as the control. In some experiments, the intestinal-specific FXR inhibitor Gly- $\beta-M C A(10 \mathrm{mg} / \mathrm{kg})$ and the global FXR antagonist Z/E-guggulsterone $(10 \mathrm{mg} / \mathrm{kg})$ were orally administered along with LR and LC for 8 weeks[12].

Probiotic preparations

LP and LR were incubated with de Man-Rogosa-Sharpe liquid medium (MRS broth, BD, USA) under anaerobic conditions at $37^{\circ} \mathrm{C}$ for 24 hours to reach the early stationary phase. The cultures were centrifuged at $5000 \times \mathrm{g}$ for $5 \mathrm{~min}$ at $4{ }^{\circ} \mathrm{C}$, and bacterial cells were resuspended in sterilized saline solution $\left(10^{9}\right.$ cells $\left./ 200 \mu \mathrm{L}\right)$ and stored at $-80^{\circ} \mathrm{C}$ as a stock solution. Each aliquot was thawed for 1 hour to room temperature before it was administered to each mouse by oral gavage. To determine the number of bacterial cells, serial dilutions of the bacterial suspensions were inoculated on fresh MRS agar plates under anaerobic conditions.

Sample collection 
The mice were treated for 8 weeks after a week of adaptation. The body weight and food intake of the mice were checked once a week[52]. At the beginning of the 8th week after the start of LP and LR administration, fecal samples were collected from these mice and stored in liquid nitrogen for RNA extraction and subsequent quantification of the strain.

At the end of the 8th week, the mice were fasted for 5 hours but allowed free access to water. Blood samples were collected by heart puncture and centrifuged at $400 \times \mathrm{g}$ for $20 \mathrm{~min}$ at $4{ }^{\circ} \mathrm{C}$ for subsequent biochemical analysis. The animals were sacrificed by exsanguination after being anaesthetized with pentobarbital. The liver, gallbladder, and ileum were harvested. Half of these tissues were immediately snap-frozen in liquid nitrogen, and the other half were fixed in formalin.

Histology

Fresh liver and gallbladder specimens were fixed in $4 \%$ neutral paraformaldehyde at room temperature for $24 \mathrm{~h}$. Paraffin-embedded sections $(4 \mu \mathrm{m})$ were stained with hematoxylin and eosin (H\&E) and assessed using a $20 \times$ objective over 5 separate fields by two expert liver pathologists who were blinded to the treatment groups to identify the percentage of hepatocytes involved by steatosis, and subsequently the steatosis score was determined using the NAS histologic scoring system.

Hepatic lipid accumulation was measured by oil red O (ORO) staining as described previously[55]. Frozen liver sections $(6 \mu \mathrm{m})$ were stained with ORO lipid stain (Abcam, USA) and assessed in the same way to identify the percentage of hepatocytes involved by steatosis.

Bile acid analysis

Ten microliters of serum was accurately measured, $300 \mu \mathrm{L}$ of ethanol was added for precipitation, and the sample was vortexed for 60 seconds and centrifuged at $12000 \times \mathrm{g}$ at $4{ }^{\circ} \mathrm{C}$ for $10 \mathrm{~min}$. The supernatants were further diluted with $100 \mu \mathrm{L}$ of methanol to reconstitute the sample and vortexed for $30 \mathrm{~s}$. Bile acid concentrations were determined using liquid chromatography-mass spectrometry (LC-MS) (Waters Corp., Milford, USA) with an electrospray ionization source. Chromatographic separation was archived on an Acquity UPLC $\circledast$ BEH C18 column (100 mm inner diameter, $1.7 \mu \mathrm{m}$; Waters Corp.). The mobile phase consisted of a mixture of $0.1 \%$ formic acid in water $(A)$ and $0.1 \%$ formic acid in acetonitrile (B). Gradient elution was applied for the following phases: $0 \sim 4 \min , 25 \%$ B; $4 \sim 9 \min , 25 \sim 30 \%$ B; $9 \sim$ $14 \mathrm{~min}, 30 \sim 36 \% \mathrm{~B} ; 14 \sim 18 \mathrm{~min}, 36 \sim 38 \% \mathrm{~B} ; 18 \sim 24 \mathrm{~min}, 38 \sim 50 \% \mathrm{~B} ; 24 \sim 32 \mathrm{~min}, 50 \sim 75 \% \mathrm{~B} ; 32$ $35 \mathrm{~min}, 75 \sim 100 \% \mathrm{~B} ; 35 \sim 38 \mathrm{~min}, 100 \sim 25 \% \mathrm{~B}$. The flow rate was $0.25 \mathrm{~mL} / \mathrm{min}$.

Immunofluorescence

The immunofluorescence of FGF15 was evaluated on paraffin-embedded lleum tissue sections. Briefly, after dewaxing and hydration, the antigen of sections was retrieved with citrate solution (Sangon Biotech (Shanghai) Co., Ltd, Shanghai, China). The sections were permeabilized in 0.3\% Triton X-100 (Sangon Biotech (Shanghai) Co., Ltd, Shanghai, China) for $10 \mathrm{~min}$, and nonspecific binding was blocked by immunostaining blocking buffer (Sangon Biotech (Shanghai) Co., Ltd, Shanghai, China) for $1 \mathrm{~h}$ at room 
temperature. Tissue sections were incubated with primary antibodies against FGF15 (1:100, Santa, USA) overnight at $4{ }^{\circ} \mathrm{C}$. After washing, the sections were incubated with goat anti-mouse or goat anti-rabbit IRDye 594 CW-labeled secondary antibodies (1:1000, Cell Signaling Technology, USA) for $1 \mathrm{~h}$ at room temperature. The nuclei were stained with DAPI ( $5 \mathrm{mg} / \mathrm{ml}$, Yeasen Biotech Co., Ltd, Shanghai, China). Finally, the sections were imaged with a Leica TCS SP8 confocal microscope.

Western blotting

Total proteins from liver and ileum tissues were extracted as previously described[56]. Total proteins (40 $\mu \mathrm{g}$ per lane) were separated by $10 \%$ SDS-PAGE and then electrotransferred to nitrocellulose membranes. The membranes were incubated with primary antibodies against FXR (NR1H4, 1:1000, Abcam, USA), SREBP2 (1:1000, Abcam, USA), CYP7A1 (1:1000, Abcam, USA), CYP8B1 (1:1000, Abcam, USA), CYP7B1 (1:1000, Proteintech Group, USA), CYP27A1 (1:1000, Proteintech Group, USA) and $\beta$-actin (1:1000, Cell Signaling Technology, USA) overnight at $4{ }^{\circ} \mathrm{C}$. After washing with $0.1 \%$ Tween 20 phosphate buffer solution (PBST) three times, the membranes were incubated with goat anti-mouse or goat antirabbit IR-Dye 700 or $800 \mathrm{CW}$-labeled secondary antibodies for 1 hour at $37^{\circ} \mathrm{C}$ and imaged with an Odyssey infrared scanner (LI-COR, Lincoln, NE, USA). Quantification was performed using the LI-COR software Image Studio.

RNA extraction and quantitative real-time polymerase chain reaction

Total RNA from liver and ileum tissues was extracted using TRIzol (Invitrogen, CA, USA) homogenization followed by isopropanol incubation as previously described, and the purity of RNA products was determined to be between 1.8 and 2.0 according to the $260 / 280$ ratio. One thousand ng of RNA was subjected to reverse transcription to generate complementary DNA using a commercial PrimeScript ${ }^{\mathrm{TM}} \mathrm{RT}$ reagent kit (Takara, Japan). The synthesized CDNA was used for quantitative polymerase chain reaction (qPCR) to determine the relative expression of targeted genes using gene-specific, intron-spanning primers (Table 1). qPCR was performed in $20 \mu \mathrm{L}$ reactions using Hieff® qPCR SYBR Green Master Mix (Yeasen Biotech, Shanghai, China) to analyze mRNA transcripts. All reactions were performed using the ABI Prism 7900HT Sequence Detection System (Applied Biosystems, CA, USA). The fold changes in the expression of each target gene were compared to the housekeeping gene $\beta$-actin using the $2^{-\triangle \triangle C T}$ method and are represented as the fold change relative to the control group. Each target gene analyzed in the tissues was analyzed in triplicate in experiments that were repeated three times. 
Table 1

PCR Genes Primers Sequences

\begin{tabular}{|c|c|}
\hline $\begin{array}{l}\text { Gene } \\
\text { (mouse) }\end{array}$ & Primer sequences \\
\hline Fxr Forward & 5'- AGCATTACCAAGAACGCCGTGTAC - 3' \\
\hline Reverse & 5'- GCTGTCGTCCTCATTAGCTGTCTG - 3' \\
\hline Fgf15 Forward & 5'- CGGTCGCTCTGAAGACGATTGC - 3' \\
\hline Reverse & 5'- TAСАТССТССАССАТССТGAACGG - 3' \\
\hline Fgfr4 Forward & 5'- GCCCCTGTACGTGATTGTGG - 3' \\
\hline Reverse & 5'- ATCCATTTGACTGGCAGGCG-3' \\
\hline Shp Forward & 5'- TCCTAGCCAAGACAGTAGCCTTCC - 3' \\
\hline Reverse & 5'- TACCGCTGCTGGCTTCCTCTAG - 3' \\
\hline Ibat Forward & 5'- АCCACTTGCTCCACACTGCTT - 3' \\
\hline Reverse & 5'- CGTTCCTGAGTCAACCCACAT - 3' \\
\hline Mrp3 Forward & 5'- TGAGATCGTCATTGATGGGC - 3' \\
\hline Reverse & 5'- AGCTGAGAGCGCAGGTCG - 3' \\
\hline Mrp4 Forward & 5'- TTAGATGGGCCTCTGGTTCT - 3' \\
\hline Reverse & 5'- GCCCACAATTCCAACCTTT - 3' \\
\hline Mdr2 Forward & 5'- GGATGGTGACTGTGGGCTGAT - 3' \\
\hline Reverse & 5'- GGCTGTTCTCCCTTCTCATGG - 3' \\
\hline Bsep Forward & 5'- GCTGCCAAGGATGCTAATGC - 3' \\
\hline Reverse & 5'- CTACCCTTTGCTTCTGCCCA - 3' \\
\hline Cyp7a1 Forward & 5'- GCTAAGACGCACCTCGTGATCC - 3' \\
\hline Reverse & 5'- CCGCAGAGCCTCCTTGATGATG - 3' \\
\hline Cyp8b1 Forward & 5'- GGCAAGAAGATCCACCACTACAGC - 3' \\
\hline Reverse & 5'- TCAGGCGATAGAGGAAGCGTACC-3' \\
\hline Cyp27a1 Forward & 5'- ATTAAGGAGACCCTGCGCCT - 3' \\
\hline Reverse & 5'- AGGCAAGACCGAACCCCATA - 3' \\
\hline Cyp7b1 Forward & 5'- TGGCTTCCTTATCTTGGCATGGC - 3' \\
\hline Reverse & 5'- TCGCTGATAATCGGCTGCTGAAC - 3' \\
\hline
\end{tabular}


Fecal samples were collected within 15 min of defecation and stored in liquid nitrogen within 1 hour. DNA extraction was performed with the QIAamp DNA Stool Kit (Qiagen, California, USA). The bacterial 16S ribosomal RNA (rRNA) gene was PCR amplified using primers binding to the V3-V4 regions. The resulting amplicons were purified by gel extraction (AxyPrep DNA GelExtraction Kit, Axygen Biosciences, Union City, California, USA) and then quantified and sequenced on the Illumina MiSeq platform (Illumina, San Diego, USA) with paired-end 300-nucleotide reads. The 16S rRNA sequencing data were analyzed by the Quantitative Insights Into Microbial Ecology platform (V.1.9.1). Sequence files and metadata for all samples used in this study have been deposited in the GenBank Sequence Read Archive database.

Bioinformatics and statistical analysis

The raw 16S rRNA gene sequencing reads were demultiplexed, quality-filtered by fastp version 0.20 .0 and merged by FLASH version 1.2.7[57, 58] with the following criteria: (1) No contaminant sequences; (2) The $300 \mathrm{bp}$ reads were truncated at any site receiving an average quality score of $<20$ over a 50 bp sliding window, and the part of truncated reads shorter than $50 \mathrm{bp}$ and the part of reads containing ambiguous characters were both discarded; (3) Only overlapping sequences longer than $10 \mathrm{bp}$ were assembled according to their overlapped sequence. The maximum mismatch ratio of overlap region is 0.2 . Reads that could not be assembled were discarded; (4) Samples were distinguished according to the barcode and primers, and the sequence direction was adjusted, exact barcode matching, 2 nucleotide mis-match in primer matching. Operational taxonomic units (OTUs) with $97 \%$ similarity cutoff[59] were clustered using UPARSE version 7.1, and chimeric sequences were identified and removed. The taxonomy of each OTU representative sequence was analyzed by RDP Classifier version 2.2 against the 16S rRNA database using confidence threshold of 0.7[60].

Principal coordinate analysis (PCOA) based on Bray-Curtis dissimilarity were performed to provide an overview of gut microbial dynamics in response to LD and LR/LP treatments. Similarities of gut microbiota between samples were compared by ANOSIM and Adonis based on Bray-Curtis in phylum level. Statistical comparison of the relative abundance in different groups were analyzed by KruskalWallis $\mathrm{H}$ test and using Welch's $\mathrm{t}$ test (with $95 \%$ confidence intervals, $P$-value $<0.05$, multiple testing $P$ values were adjusted with FDR). The relationship between the top 50 abundance of Species levels and the incidence and grade of CGS and the serum BAs, the serum biochemical values and the CGS-related mRNA expressions were analyzed by Spearman's correlation analysis, the Spearman's correlation coefficient $|\mathrm{R}| \geq 0.1$ and $* 0.01<P \leq 0.05, * \star 0.001<P \leq 0.01$, *** $P \leq 0.001$.

Reagents

Z/E-Guggulsterone was purchased from MCE (MCE, USA). Primary antibodies against CYP7A1, CYP8B1 and FXR were purchased from Abcam (Abcam, USA). $\beta$-actin was purchased from Cell Signaling Technology (CST, USA). The primary antibodies against CYP7B1, CYP27A1 and FGFR4 were from 
Proteintech Group (Proteintech Group, USA). The primary antibodies against FGF15 and all the other reagents were from Sigma-Aldrich Chemical (MO, USA).

\section{Statistical analysis}

Data were presented as the mean \pm SEM. Statistical analysis was performed using GraphPad Prism 7.0 (GraphPad, La Jolla, CA). Two groups were compared by an unpaired t-test. A P value $<0.05$ was considered statistically significant.

\section{Abbreviations}

CGS, Cholesterol Gallstone, BA, bile acid, LR, Lactobacillus reuteri CGMCC 17942, LP, L. plantarum CGMCC 14407, T-a-MCA, taurine-a-muricholic acid, T- $\beta$-MCA, taurine-- $\beta$-muricholic acid, FXR, Farnesoid X Receptor, FGF15, fibroblast growth factor 15, SHP, small heterodimer partner, CYP7A1, cholesterol 7ahydroxylase, CYP7B1, oxysterol 7a-hydroxylase, CYP8B1, Sterol 12a-hydroxylase, CYP27A1, cholesterol 27a-hydroxylase, MDR2, multidrug resistance protein, MRP, multidrug-resistance-associated protein homologues, BSEP, bile salt export pump, IBAT, ileal bile acid transporter, LD, lithogenic diet, ND, normal diet, CA, cholic acid, CDCA, chenodeoxycholic acid, TCDCA, taurochenodeoxycholic acid, Z-Gu, (Z)Guggulsterone, Gly-MCA, glycine- $\beta$-muricholic acid.

\section{Declarations}

\section{Availability of data and materials}

The sequence files and metadata for all samples used in this study have been deposited into the NCBI Sequence Read Archive (SRA) database (http://www.ncbi.nlm.nih.gov/bioproject/680858).

\section{Funding}

This work was sponsored by National Natural Science Foundation of China to X.W. (81870452), J.W (81600409) and The Action Plan for Scientific and Technological Innovation Program from the Shanghai Science and Technology Committee (19411951500) and Shanghai Songjiang district science and technology project (0702N17002) and Shanghai General Hospital to J.W (06N1702003).

\section{Author contributions}

X.W. and J.W. designed, conceived and supervised the study. X.W. and J.W. provided funding to support the study. X.Y. and Q.Z. performed the experiments. X.Y. collected and analyzed the data. Z.D., X.W. and J.X. provided technical support in the in vivo experiments. S.W., M.N.and S.S drafted the manuscript. X.W. and J.W. revised the manuscript. All the authors approved the final version of the manuscript.

\section{Correspondence:}


Dr. Xinjian Wan, Ph.D., M.D. (slwanxinjian2020@126.com), Department of Gastroenterology, Shanghai Sixth People's Hospital, Shanghai Jiao Tong University, China.

Dr. Jingjing Wang, Ph.D. (wangjingjing6891@163.com)

Shanghai Key Laboratory of Pancreatic Disease, Shanghai General Hospital, Shanghai Jiao Tong University School of Medicine, 100 Haining Road, Shanghai 200080, China. Tel: (+86)021-632400903151.

\section{Acknowledgement}

The authors would like to express their gratitude to American Journal Experts (https://secure.aje.com) for the expert linguistic services provided.

\section{Ethics declarations}

Ethics approval and consent to participate

See animal paragraph in the "Materials and methods" section.

\section{Consent for publication}

Not applicable

Conflict of Interest: The authors have declared that there is no conflict of interest.

\section{References}

1. Forrest $\mathrm{K}$, Welch $\mathrm{C}$, Williams $\mathrm{E}, \mathrm{Smart} \mathrm{H}$, Lombard M. Investigation of cholesterol gallstone disease. Lancet. 2006;368:989-90.

2. Lammert F, Gurusamy K, Ko CW, Miquel J-F, Méndez-Sánchez N, Portincasa P, et al. Gallstones. Nat Rev Dis Primers. 2016;2:16024.

3. Wang J, Xu C, Cheng Q, Zhao J, Wu S, Li W, et al. RNA Sequencing Revealed Signals of Evolution From Gallbladder Stone to Gallbladder Carcinoma. Front Oncol. 2020;10:823.

4. Shabanzadeh DM, Sørensen LT, Jørgensen T. Association Between Screen-Detected Gallstone Disease and Cancer in a Cohort Study. Gastroenterology. 2017;152:1965-1974.e1.

5. Grigor'eva IN, Romanova TI. Gallstone Disease and Microbiome. Microorganisms [Internet]. 2020 [cited 2020 Aug 11];8. Available from: https://www.ncbi.nlm.nih.gov/pmc/articles/PMC7356158/

6. Wells JE, Berr F, Thomas LA, Dowling RH, Hylemon PB. Isolation and characterization of cholic acid 7a-dehydroxylating fecal bacteria from cholesterol gallstone patients. Journal of Hepatology. 2000;32:4-10. 
7. Wang Q, Hao C, Yao W, Zhu D, Lu H, Li L, et al. Intestinal flora imbalance affects bile acid metabolism and is associated with gallstone formation. BMC Gastroenterol 2020;20(1):59

8. Wang Q, Jiao L, He C, Sun H, Cai Q, Han T, et al. Alteration of gut microbiota in association with cholesterol gallstone formation in mice. BMC Gastroenterol. 2017;17:74.

9. Takeda Y, Itoh H, Kobashi K. EFFECT of CLOSTRIDIUM BUTYRICUM ON THE FORMATION AND DISSOLUTION OF GALLSTONES IN EXPERIMENTAL CHOLESTEROL CHOLELITHIASIS. 1983;32:6.

10. Molinero N, Ruiz L, Sánchez B, Margolles A, Delgado S. Intestinal Bacteria Interplay With Bile and Cholesterol Metabolism: Implications on Host Physiology. Front Physiol. 2019;10:185.

11. Yu Z, Shi Z, Zheng Z, Han J, Yang W, Lu R, et al. DEHP induce cholesterol imbalance via disturbing bile acid metabolism by altering the composition of gut microbiota in rats. Chemosphere. 2020;263:127959.

12. Liu Y, Chen K, Li F, Gu Z, Liu Q, He L, et al. Probiotic Lactobacillus rhamnosus GG Prevents Liver Fibrosis Through Inhibiting Hepatic Bile Acid Synthesis and Enhancing Bile Acid Excretion in Mice. Hepatology. 2020;71:2050-66.

13. Tarling EJ, Ahn H, de Aguiar Vallim TQ. The nuclear receptor FXR uncouples the actions of miR-33 from SREBP-2. Arterioscler Thromb Vasc Biol. 2015;35:787-95.

14. Cai J, Wang Z, Chen G, Li D, Liu J, Hu H, et al. The reabsorption of bile acids regulated by FXROATP1A2 is the main factor for the formation of cholesterol gallstone. Am J Physiol Gastrointest Liver Physiol. 2020;

15. Moschetta A, Bookout AL, Mangelsdorf DJ. Prevention of cholesterol gallstone disease by FXR agonists in a mouse model. Nat Med. 2004;10:1352-8.

16. Li F, Jiang C, Krausz KW, Li Y, Albert I, Hao H, et al. Microbiome remodelling leads to inhibition of intestinal farnesoid $X$ receptor signalling and decreased obesity. Nature Communications. 2013;4:2384.

17. Moschetta A, Bookout AL, Mangelsdorf DJ. Prevention of cholesterol gallstone disease by FXR agonists in a mouse model. Nat Med. 2004;10:1352-8.

18. Wang G, Huang W, Xia Y, Xiong Z, Ai L. Cholesterol-lowering potentials of Lactobacillus strain overexpression of bile salt hydrolase on high cholesterol diet-induced hypercholesterolemic mice. Food Funct. 2019;10:1684-95.

19. Reis SA, Conceição LL, Rosa DD, Siqueira NP, Peluzio MCG. Mechanisms responsible for the hypocholesterolaemic effect of regular consumption of probiotics. Nutr Res Rev. 2017;30:36-49.

20. Akiyoshi T, Uchida K, Takase H, Nomura Y, Takeuchi N. Cholesterol gallstones in alloxan-diabetic mice. J Lipid Res. 1986;27:915-24.

21. Li YJ, Jun HJ, Ning C, Sheng ZD, Min LS, Ya HW, et al. Sex-specific and Dose-response Relationship between the Incidence of Gallstones and Components of the Metabolic Syndrome in Jinchang Cohort: A Prospective Study. BES. Biomedical and Environmental Sciences; 2020;33:633-8. 
22. Nervi F, Miquel JF, Alvarez M, Ferreccio C, García-Zattera MJ, González R, et al. Gallbladder disease is associated with insulin resistance in a high risk Hispanic population. J Hepatol. 2006;45:299-305.

23. Biddinger SB, Haas JT, Yu BB, Bezy O, Jing E, Zhang W, et al. Hepatic insulin resistance directly promotes formation of cholesterol gallstones. Nat Med. 2008;14:778-82.

24. Alvaro D. Gallstones: Bad Company for the Steatotic Liver. Gastroenterology. 2017;152:1284-6.

25. Yang F, Mao C, Guo L, Lin J, Ming Q, Xiao P, et al. Structural basis of GPBAR activation and bile acid recognition. Nature. 2020;

26. Funabashi M, Grove TL, Wang M, Varma Y, McFadden ME, Brown LC, et al. A metabolic pathway for bile acid dehydroxylation by the gut microbiome. Nature. 2020;582:566-70.

27. Chen J, Thomsen M, Vitetta L. Interaction of gut microbiota with dysregulation of bile acids in the pathogenesis of nonalcoholic fatty liver disease and potential therapeutic implications of probiotics. Journal of Cellular Biochemistry. 2019;120:2713-20.

28. Chiang JYL, Ferrell JM. Bile Acid Metabolism in Liver Pathobiology. Gene Expr. 2018;18:71-87.

29. Chiang JYL. Targeting bile acids and lipotoxicity for NASH treatment. Hepatology Communications. 2017;1:1002-4.

30. Jia W, Xie G, Jia W. Bile acid-microbiota crosstalk in gastrointestinal inflammation and carcinogenesis. Nature Reviews Gastroenterology \& Hepatology. 2018;15:111-28.

31. Kamath BM, Stein P, Houwen RHJ, Verkade HJ. Potential of ileal bile acid transporter inhibition as a therapeutic target in Alagille syndrome and progressive familial intrahepatic cholestasis. Liver Int. 2020;

32. Müller O, Schalla C, Scheibner J, Stange EF, Fuchs M. Expression of liver plasma membrane transporters in gallstone-susceptible and gallstone-resistant mice. Biochem J. 2002;361:673-9.

33. Chai J, Cai S-Y, Liu X, Lian W, Chen S, Zhang L, et al. Canalicular membrane MRP2/ABCC2 internalization is determined by Ezrin Thr567 phosphorylation in human obstructive cholestasis. J Hepatol. 2015;63:1440-8.

34. Rius M, Hummel-Eisenbeiss J, Hofmann AF, Keppler D. Substrate specificity of human ABCC4 (MRP4)-mediated cotransport of bile acids and reduced glutathione. Am J Physiol Gastrointest Liver Physiol. 2006;290:G640-649.

35. Juran BD, Lazaridis KN. Is the FXR the fix for cholesterol gallstone disease? Hepatology (Baltimore, Md). 2005;42:218-21.

36. Chen Y, Zhou J, Xu S, Liu M, Wang M, Ma Y, et al. Association between the perturbation of bile acid homeostasis and valproic acid-induced hepatotoxicity. Biochem Pharmacol. 2019;170:113669.

37. Reihnér E, Björkhem I, Angelin B, Ewerth S, Einarsson K. Bile acid synthesis in humans: regulation of hepatic microsomal cholesterol 7 alpha-hydroxylase activity. Gastroenterology. 1989;97:1498-505.

38. Dikkers A, Freak de Boer J, Annema W, Groen AK, Tietge UJF. Scavenger receptor BI and ABCG5/G8 differentially impact biliary sterol secretion and reverse cholesterol transport in mice. Hepatology. 2013;58:293-303. 
39. Guo C, Chi Z, Jiang D, Xu T, Yu W, Wang Z, et al. Cholesterol Homeostatic Regulator SCAP-SREBP2 Integrates NLRP3 Inflammasome Activation and Cholesterol Biosynthetic Signaling in Macrophages. Immunity. 2018;49:842-856.e7.

40. Eberl G, Vieira P. Beware of whom you live with: Your intestinal IgA may depend on it. European Journal of Immunology. 2020;50:779-82.

41. Ley RE, Turnbaugh PJ, Klein S, Gordon JI. Microbial ecology: human gut microbes associated with obesity. Nature. 2006;444:1022-3.

42. Lee KS, Palatinszky M, Pereira FC, Nguyen J, Fernandez VI, Mueller AJ, et al. An automated Ramanbased platform for the sorting of live cells by functional properties. Nature Microbiology. 2019;4:1035-48.

43. Lagkouvardos I, Lesker TR, Hitch TCA, Gálvez EJC, Smit N, Neuhaus K, et al. Sequence and cultivation study of Muribaculaceae reveals novel species, host preference, and functional potential of this yet undescribed family. Microbiome. 2019;7:28.

44. Basolo A, Hohenadel M, Ang QY, Piaggi P, Heinitz S, Walter M, et al. Effects of underfeeding and oral vancomycin on gut microbiome and nutrient absorption in humans. Nature Medicine. 2020;26:58998.

45. Dao MC, Everard A, Aron-Wisnewsky J, Sokolovska N, Prifti E, Verger EO, et al. Akkermansia muciniphila and improved metabolic health during a dietary intervention in obesity: relationship with gut microbiome richness and ecology. Gut. 2016;65:426-36.

46. Zhang J, Zhang Q, Liu G, Zhang N. Therapeutic potentials and mechanisms of the Chinese traditional medicine Danshensu. European Journal of Pharmacology. 2019;864:172710.

47. Zou B, Yang W, Tang Y, Hou Y, Tang T, Qu S. Intestinal microbiota-farnesoid X receptor axis in metabolic diseases. Clin Chim Acta. 2020;509:167-71.

48. Molinero N, Ruiz L, Milani C, Gutiérrez-Díaz I, Sánchez B, Mangifesta M, et al. The human gallbladder microbiome is related to the physiological state and the biliary metabolic profile. Microbiome. 2019;7:100.

49. Zhang Z, Zhou H, Guan M, Zhou X, Liang X, Lv Y, et al. Lactobacillus casei YRL577 combined with plant extracts reduce markers of non-alcoholic fatty liver disease in mice. Br J Nutr. 2020;1-31.

50. Kim I, Ahn S-H, Inagaki T, Choi M, Ito S, Guo GL, et al. Differential regulation of bile acid homeostasis by the farnesoid X receptor in liver and intestine. J Lipid Res. 2007;48:2664-72.

51. Wang Q, Hao C, Yao W, Zhu D, Lu H, Li L, et al. Intestinal flora imbalance affects bile acid metabolism and is associated with gallstone formation. BMC Gastroenterol [Internet]. 2020 [cited 2020 May 12];20. Available from: https://www.ncbi.nlm.nih.gov/pmc/articles/PMC7060658/

52. Wang J, Tang H, Zhang C, Zhao Y, Derrien M, Rocher E, et al. Modulation of gut microbiota during probiotic-mediated attenuation of metabolic syndrome in high fat diet-fed mice. ISME J. 2015;9:115.

53. Depommier C, Everard A, Druart C, Plovier H, Van Hul M, Vieira-Silva S, et al. Supplementation with Akkermansia muciniphila in overweight and obese human volunteers: a proof-of-concept exploratory 
study. Nat Med. 2019;25:1096-103.

54. Amigo L, Castro J, Miquel JF, Zanlungo S, Young S, Nervi F. Inactivation of hepatic microsomal triglyceride transfer protein protects mice from diet-induced gallstones. Gastroenterology. 2006;131:1870-8.

55. Levene AP, Kudo H, Armstrong MJ, Thursz MR, Gedroyc WM, Anstee QM, et al. Quantifying hepatic steatosis - more than meets the eye. Histopathology. 2012;60:971-81.

56. Ye X, Han X, Li B, Dai J, Wu Z, He Y, et al. Dopamine D2 receptor activator quinpirole protects against trypsinogen activation during acute pancreatitis via upregulating HSP70. Am J Physiol Gastrointest Liver Physiol. 2020;318:G1000-12.

57. Chen S, Zhou Y, Chen Y, Gu J. fastp: an ultra-fast all-in-one FASTQ preprocessor. Bioinformatics. 2018;34:i884-90.

58. Magoč T, Salzberg SL. FLASH: fast length adjustment of short reads to improve genome assemblies. Bioinformatics. 2011;27:2957-63.

59. Edgar RC. UPARSE: highly accurate OTU sequences from microbial amplicon reads. Nat Methods. 2013;10:996-8.

60. Wang Q, Garrity GM, Tiedje JM, Cole JR. Naive Bayesian classifier for rapid assignment of rRNA sequences into the new bacterial taxonomy. Appl Environ Microbiol. 2007;73:5261-7.

\section{Figures}


A

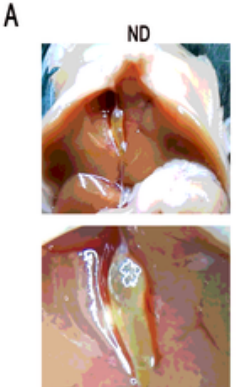

D

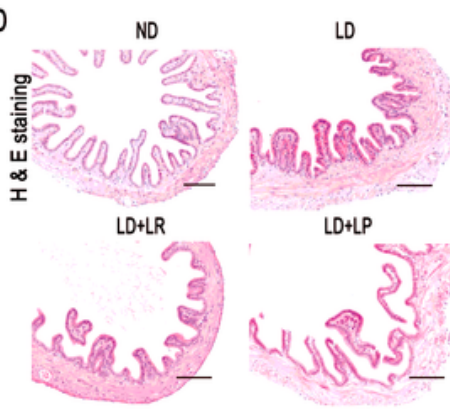

B

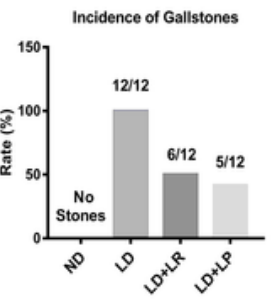

F
E

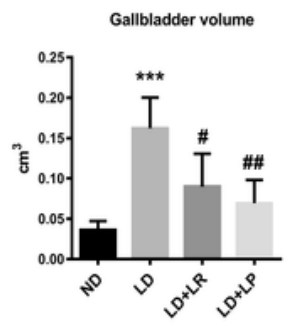

C

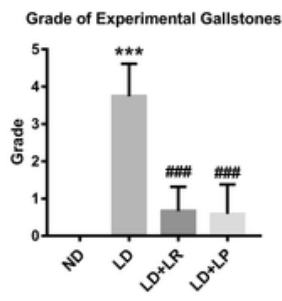

G

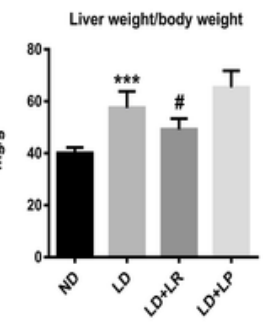

H
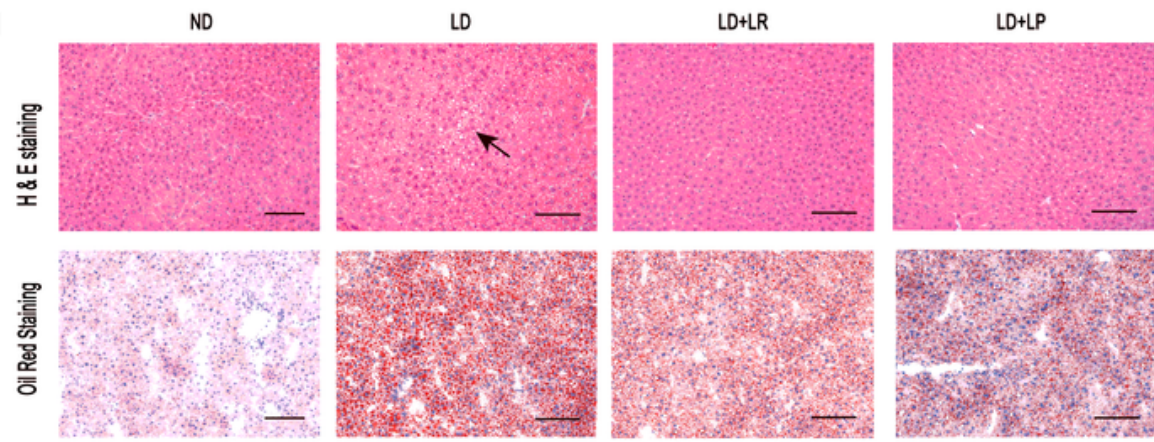

I

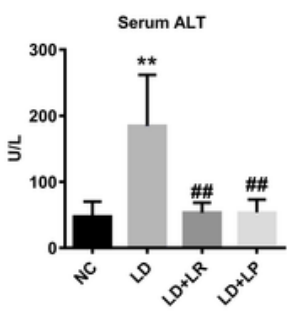

L

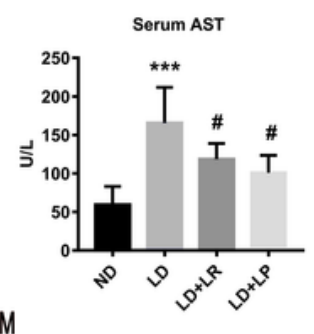

J

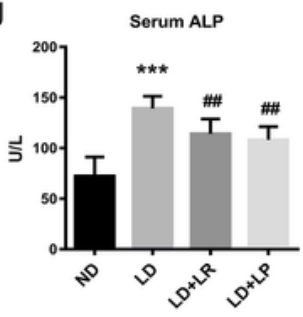

K
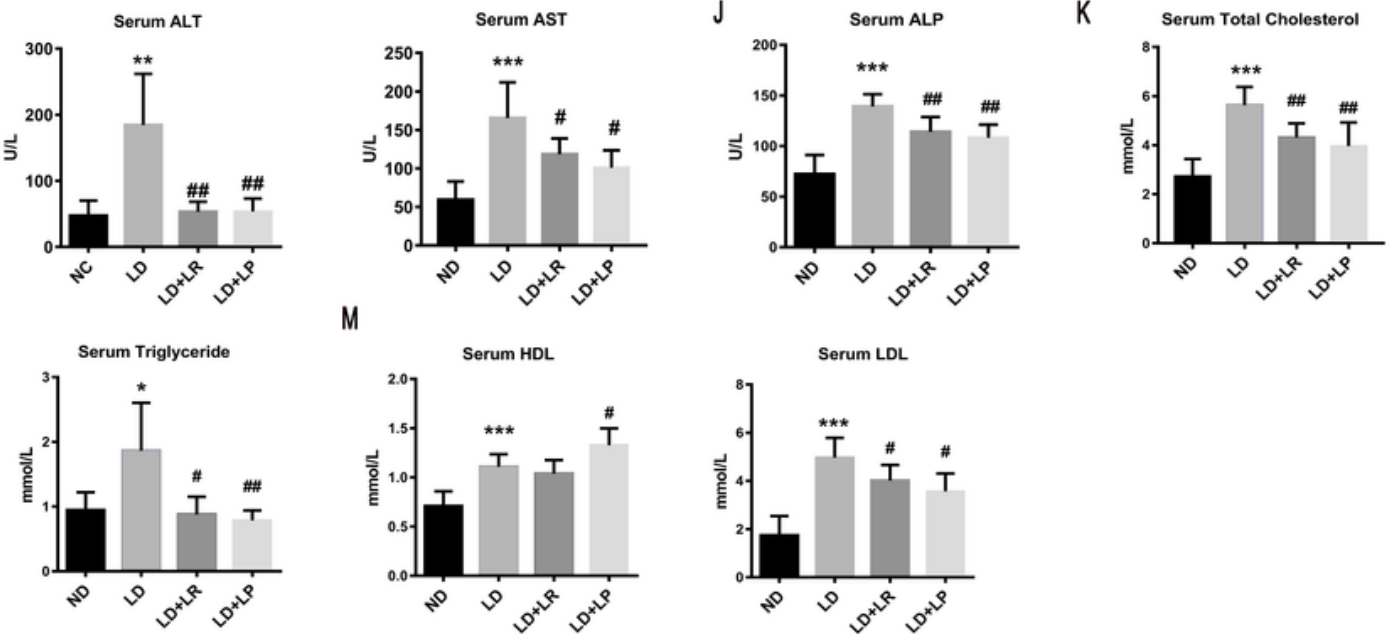

Serum LDL

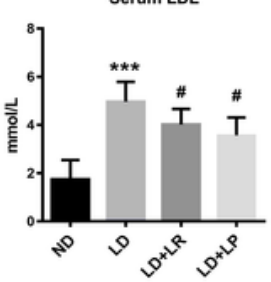

\section{Figure 1}

LR and LP treatments reduced LD-induced gallstones and metabolic disorders in mice. Twelve mice were randomly assigned to each group fed a normal diet (ND) and fed a lithogenic diet (LD) with or without LR or LP treatment (109 CFU/day) for 8 weeks. (A) Gross appearance of gallbladders and gallstones of mice administered different treatments. (B) Percentage of gallstone incidence in each group of mice. (C) The grade of experimental cholesterol gallstones (CGSs) in the mice was based on the observed 
cholelithiasis. (D) Representative images of H\&E-stained gallbladder sections (×200). (E) Gallbladder volume was estimated by the length, diameter and circumference of the gallbladder. $(F)$ Body weight of mice was recorded once a week. $(G)$ The ratio of liver weight to body weight. $(H)$ Representative images of H\&E-stained and Oil red-stained liver sections (×200). (I-M) Serum alanine aminotransferase (ALT), aspartate aminotransferase (AST), alkaline phosphatase (ALP), total cholesterol (TC), triglyceride (TG), high-density lipoprotein $(\mathrm{HDL})$ and low-density lipoprotein $(\mathrm{LDL})$ were determined by a SIEMENS fast automatic biochemical analyzer (ADVIA 2400). Data are presented as the mean \pm SEM from at least three independent experiments $(n=12), * 0.01<P \leq 0.05, * \star 0.001<P \leq 0.01$, *** P $\leq 0.001$ vs ND, $\# 0.01<P$ $\leq 0.05$, \#\# $0.001<P \leq 0.01$, \#\#\# $\leq 0.001$ vs LD. 

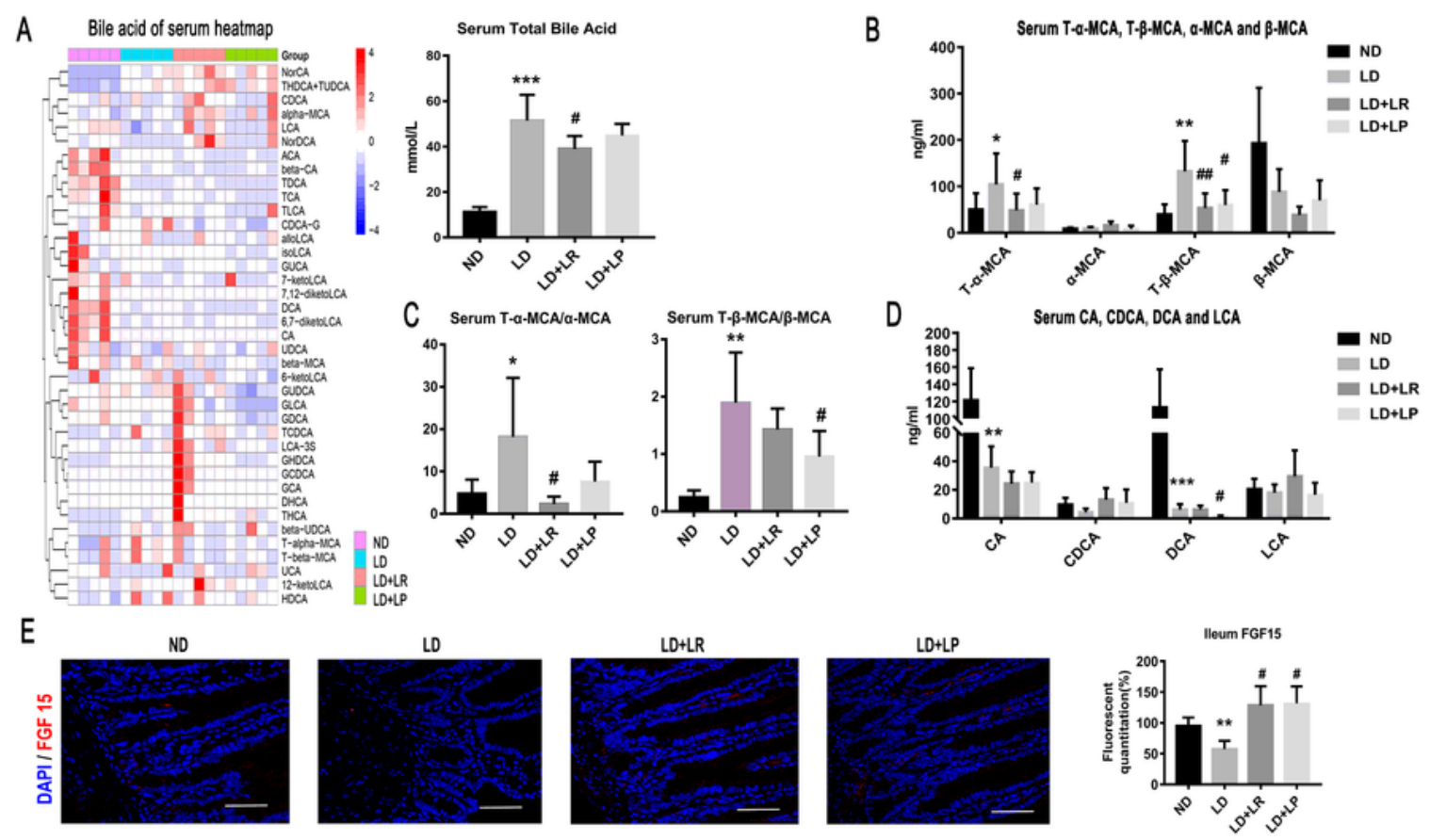

F

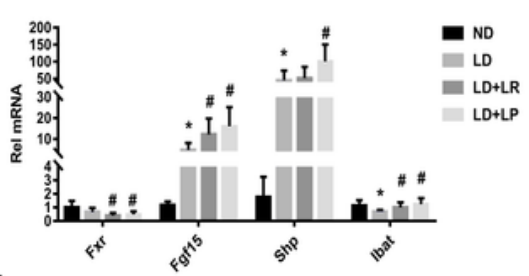

G
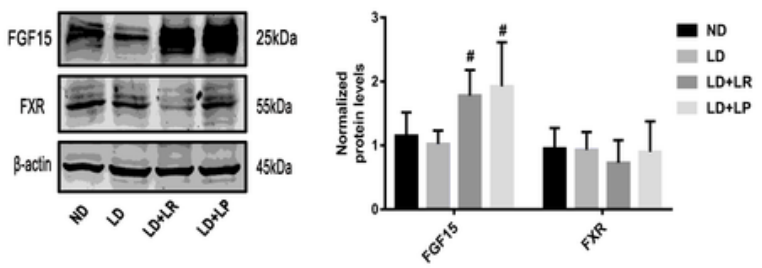

H
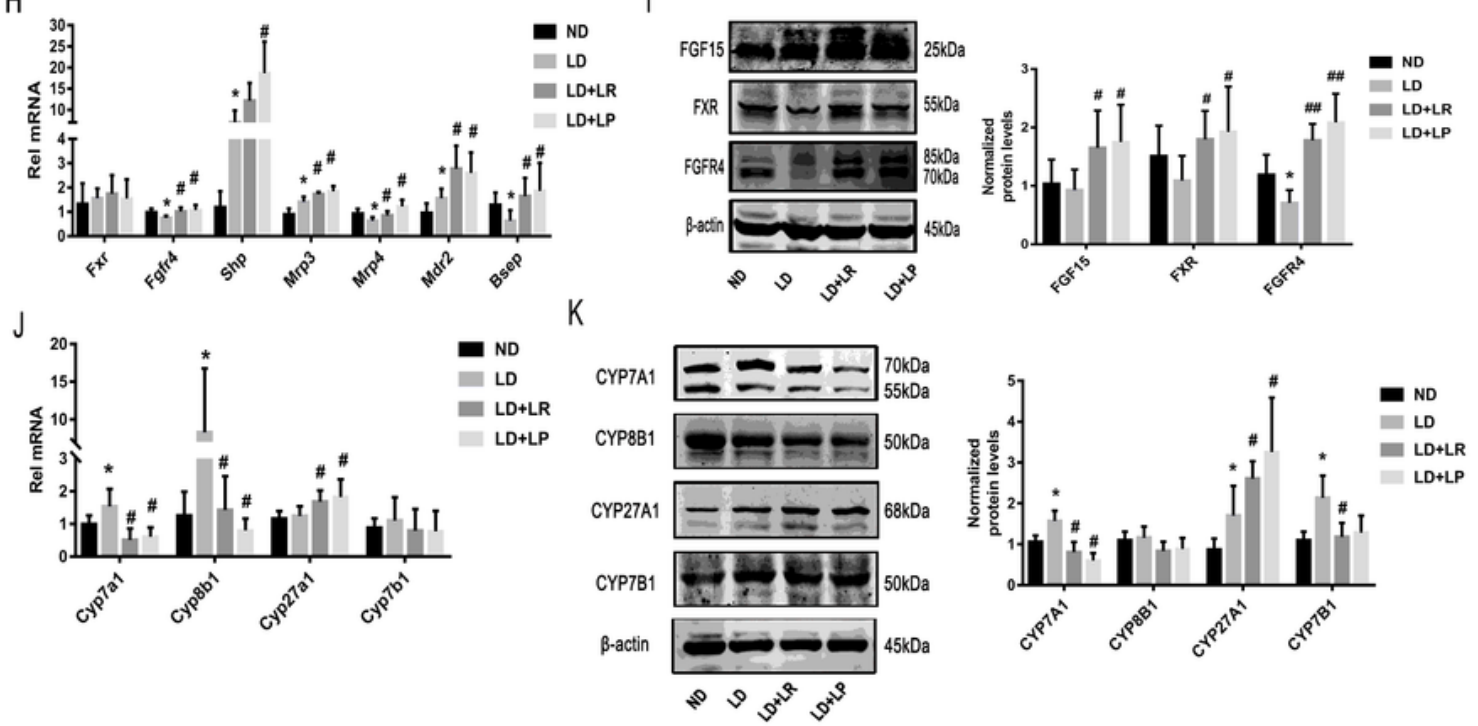

Figure 2

LR and LP treatments changed the BA composition in serum and activated FXR-FGF-15 signaling. The mice in each group were sacrificed at the end of the eighth week, and serum and tissues were collected. (A) Heatmap of the contents of 39 kinds of BAs and total bile acid (TBA) in the serum of mice in each group. The color key represents the calibration content of BAs, and the similar changes in BAs were clustered with the dendrogram on the left. (B-D) Serum T-a-MCA, T- $\beta-M C A, a-M C A, \beta-M C A, C A, C D C A, D C A$ 
and LCA were detected by high-performance liquid chromatography-tandem mass spectrometry, and the rates of T- $\alpha-M C A / \alpha-M C A$ and T- $\beta-M C A / \beta-M C A$ were calculated. (E) Immunofluorescence analyses of ileal FGF-15 ( $\times 400)$. (F) Ileal mRNA expression of Fxr, Fgf15, Shp and Ibat. (G) Protein expression and quantification of ileal FXR and FGF15. (H) Hepatic mRNA levels of Fxr, Fgfr4, Shp, Mrp3, Mrp4, Mdr2 and Bsep. (I) Protein expression and quantification of liver FGF15, FXR and FGFR4. (J) Hepatic mRNA expression of Cyp7a1, Cyp8b1, Cyp27a1, and Cyp7b1. (K) Hepatic protein expression and quantification of CYP7A1, CYP8B1, CYP27A1, and CYP7B1. Data are presented as the mean \pm SEM from at least three independent experiments $(n=6), * 0.01<P \leq 0.05$, ** $0.001<P \leq 0.01$, *** $P \leq 0.001$ vs ND, $\# 0.01<P \leq$

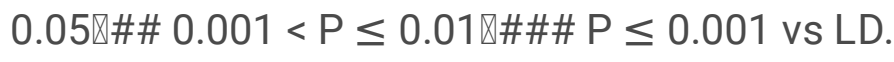


A

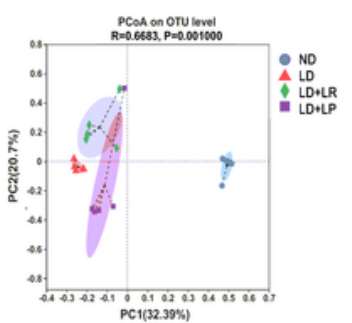

B

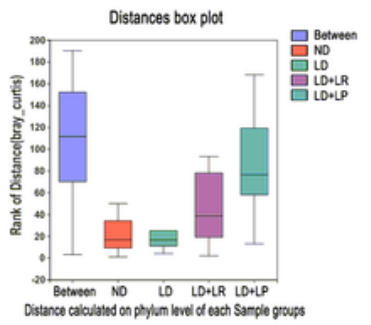

D

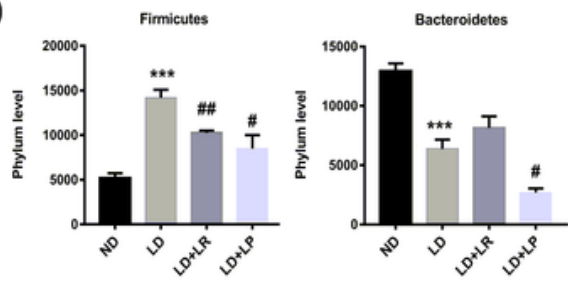

E

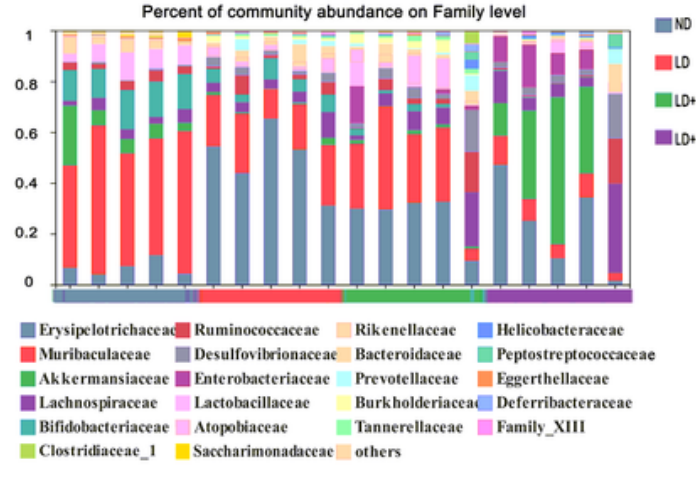

F

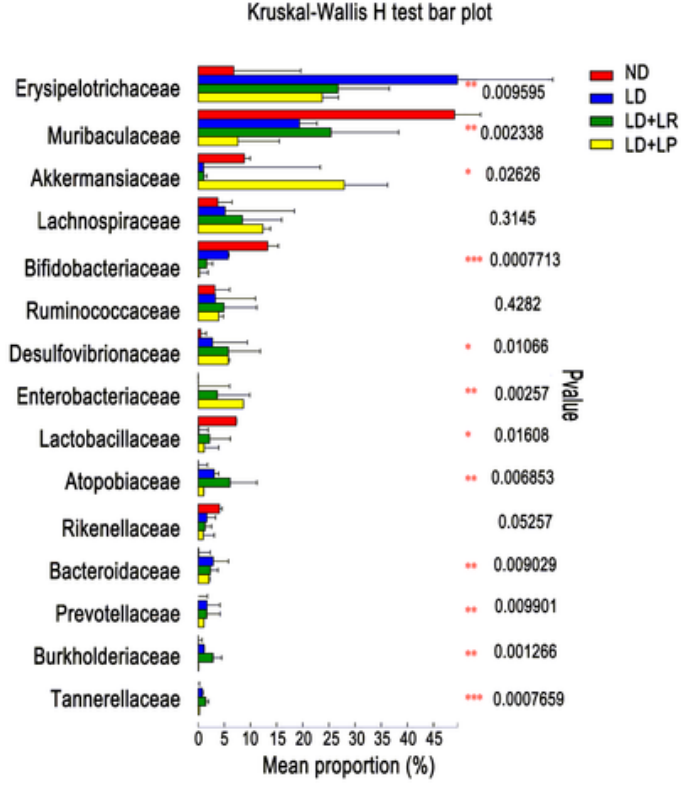

C

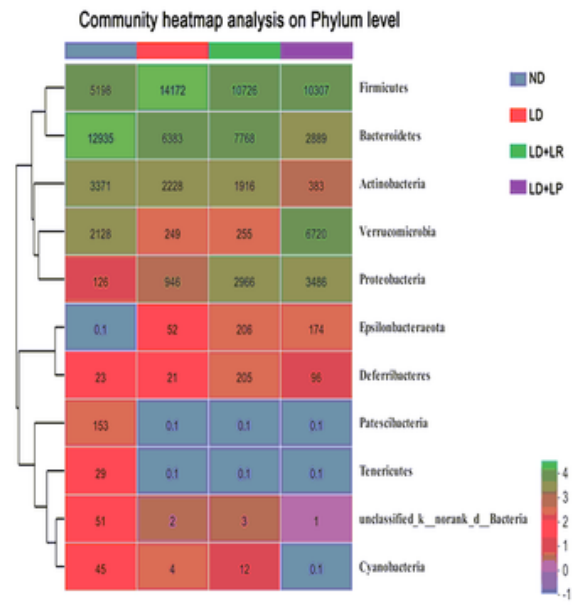

G

Community heatmap analysis on Species level

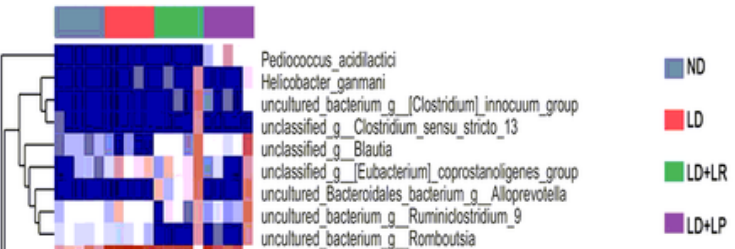

DLD+LP

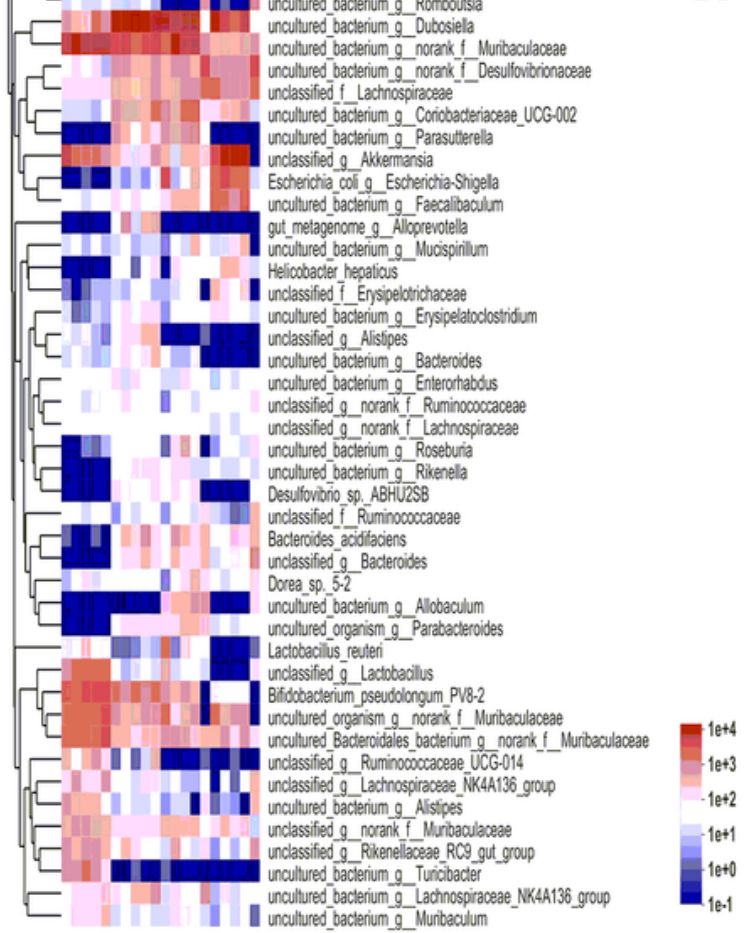

\section{Figure 3}

LR and LP treatments changed the CGS-associated gut microbiota composition of LD-fed mice. Fecal samples from ND-fed mice or LD-fed mice with or without Lactobacillus treatment were collected at the 8th week for quantification. (A) Principal coordinate analysis (PCoA) of unweighted UniFrac analysis based on the OTU abundance of different groups (Bray-Curtis ANOSIM, R2=0.6683, P=0.001, ANOSIM). (B) Distance box plot of groups (Bray-Curtis ANOSIM, R=0.6007, $P<0.001$ ). (C) Relative abundance of 
phyla in the gut microbiota of ND-fed mice and LD-fed mice with or without LR or LP treatment. (D) The abundance of Firmicutes and Bacteroidetes and the Firmicutes/Bacteroidetes (F/B) ratio. (E) The relative abundance of fecal bacteria in each group of mice at the family level was analyzed with community bar plot analysis. (F) Comparison of the top 15 most abundant families in the ND, LD, LD+LR and LD+LP groups by Kruskal-Wallis $\mathrm{H}$ test. $(\mathrm{G})$ The top 50 most abundant species in the gut microbiota in different groups are presented with a heatmap. Data are presented as the mean \pm SEM from at least three independent experiments $\left.(n=5),{ }^{*} 0.01<P \leq 0.05 \rrbracket^{\star *} 0.001<P \leq 0.01 \rrbracket^{\star \star * *} P \leq 0.001\right)$.

A

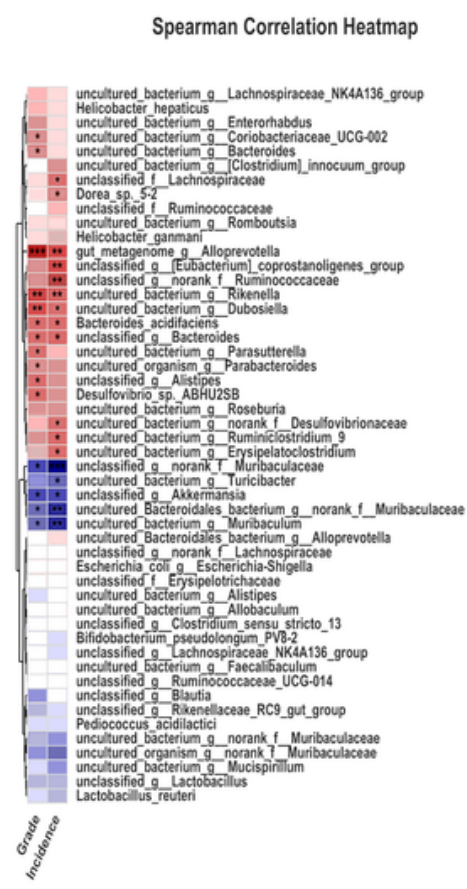

C

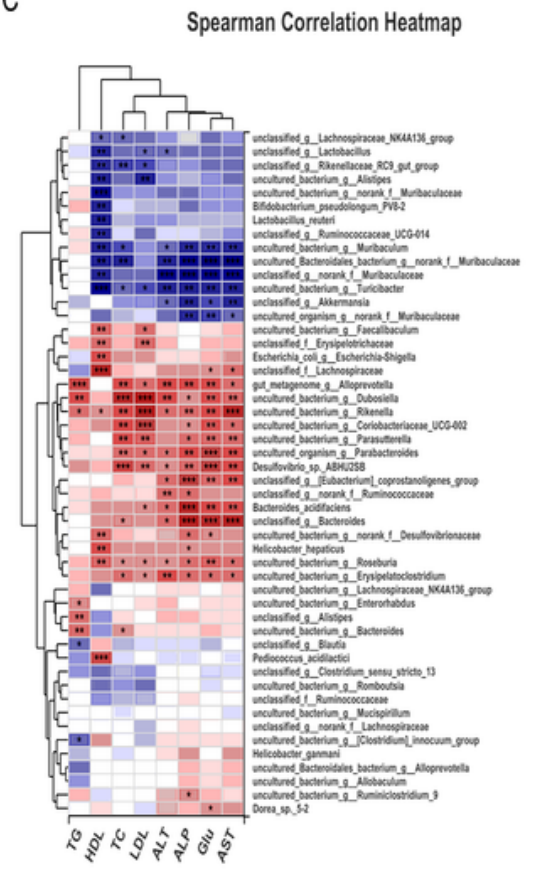

B
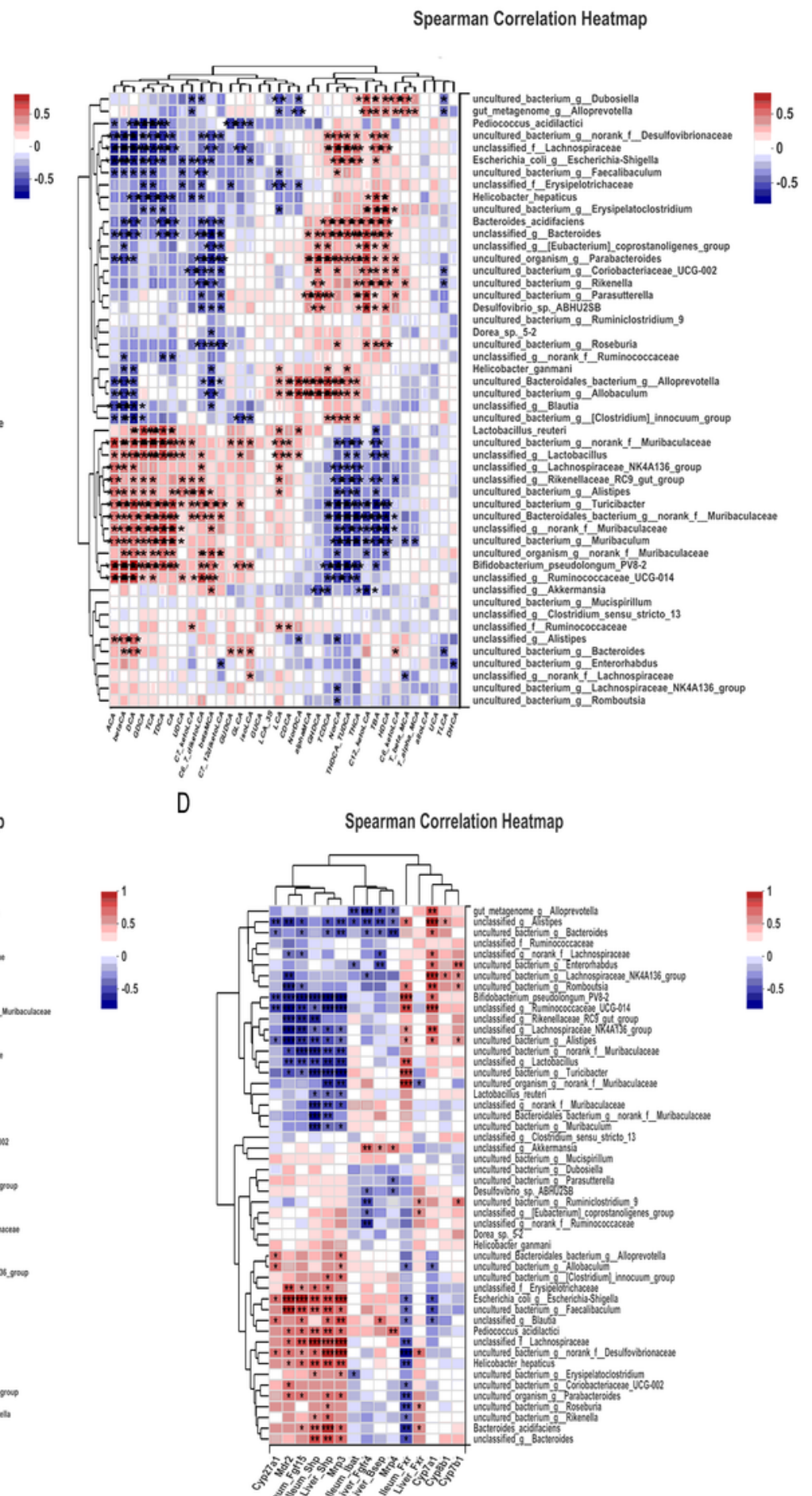

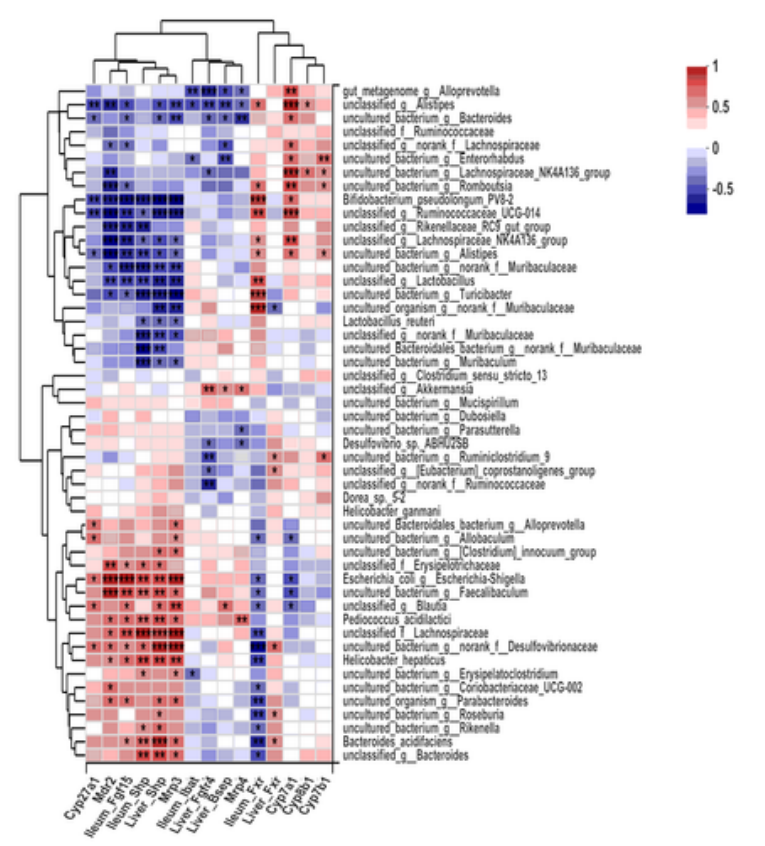




\section{Figure 4}

Correlation between gut microbiota and host CGS-related parameters. (A) Spearman's correlation analysis was performed between the top 50 most abundant species levels and the incidence and grade of CGS.

(B) Spearman's correlation analysis was performed between the top 50 most abundant species levels and the total bile acid (TBA) and 39 kinds of BAs. (C) Spearman's correlation analysis was performed between the top 50 most abundant species levels and the serum AST, ALT, ALP, TG, TC, HDL, LDL and glucose (Glu) levels. (D) Spearman's correlation analysis was performed between the top 50 most abundant species levels and the mRNA levels of liver Cyp7a1, Cyp8b1, Cyp7b1, Cyp 27a1, Mdr2, Mrp3, Mrp4, Bsep, Shp, Fxr, and Fgfr4 and ileum Fxr, Fgf15, and Shp. Red and blue denote positive and negative associations, respectively. Data are presented as the mean \pm SEM from at least three independent experiments $(n=5), * 0.01<P \leq 0.05, * \star 0.001<P \leq 0.01$, *** $P \leq 0.001,|R| \geq 0.1$. 
A

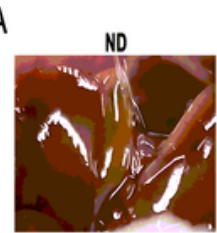

$L D+L R+Z \cdot G u$

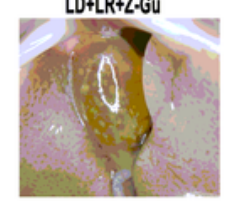

F
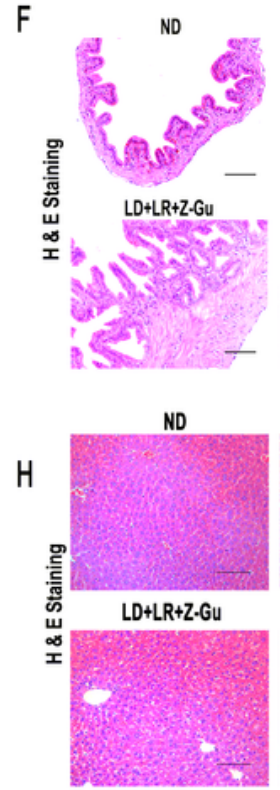

ND

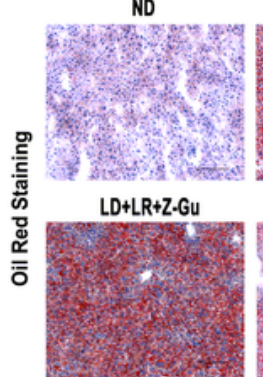

L

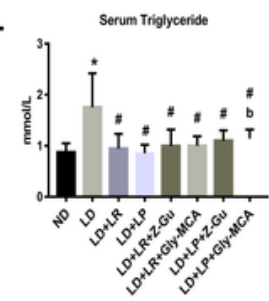

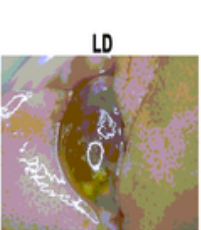

LD+LR+Gly-MCA
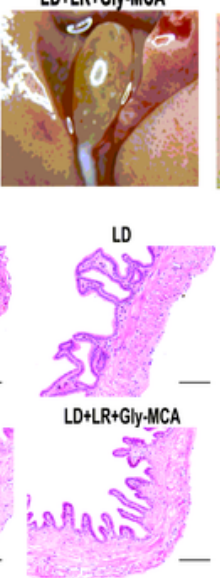

LD

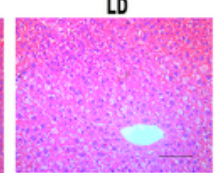

$L D+L R+G l y-M C A$

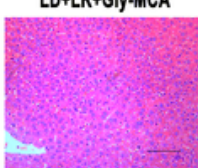

LD

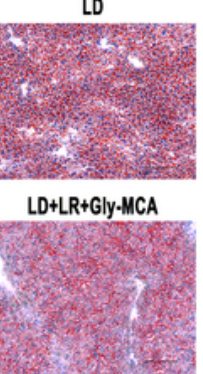

M

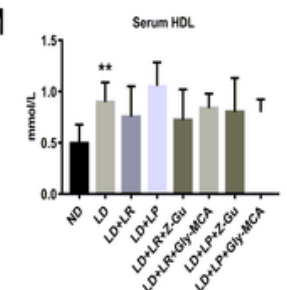

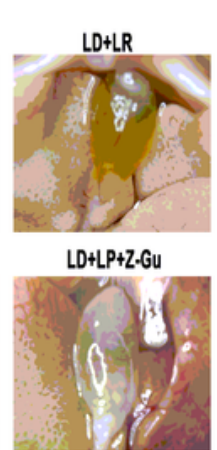

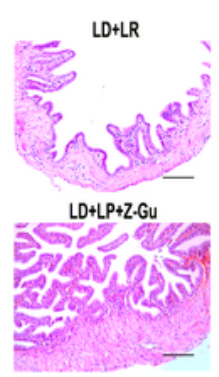

LD+LR

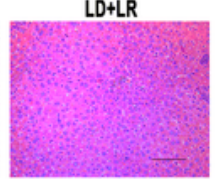

$L D+L P+Z-G u$

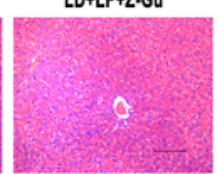

LD+LR

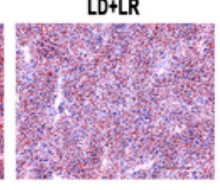

$L D+L P+Z-G u$
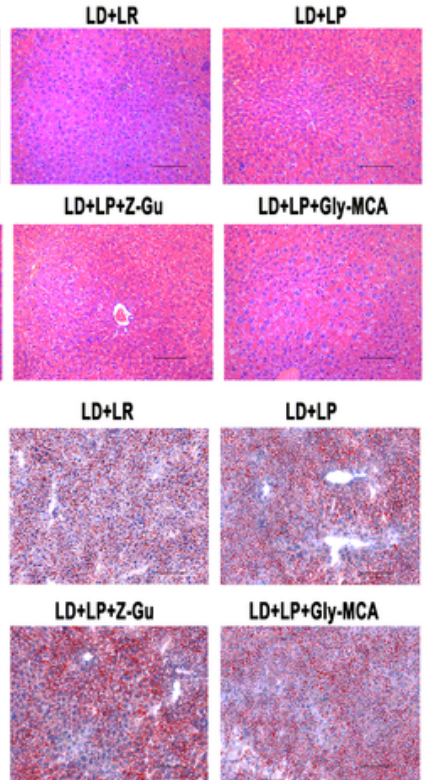

$L D+L P$

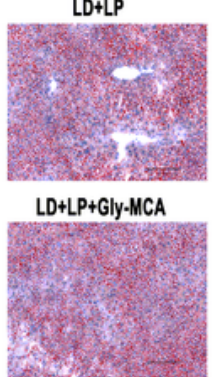

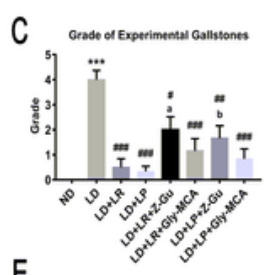

E

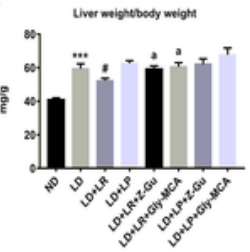

G
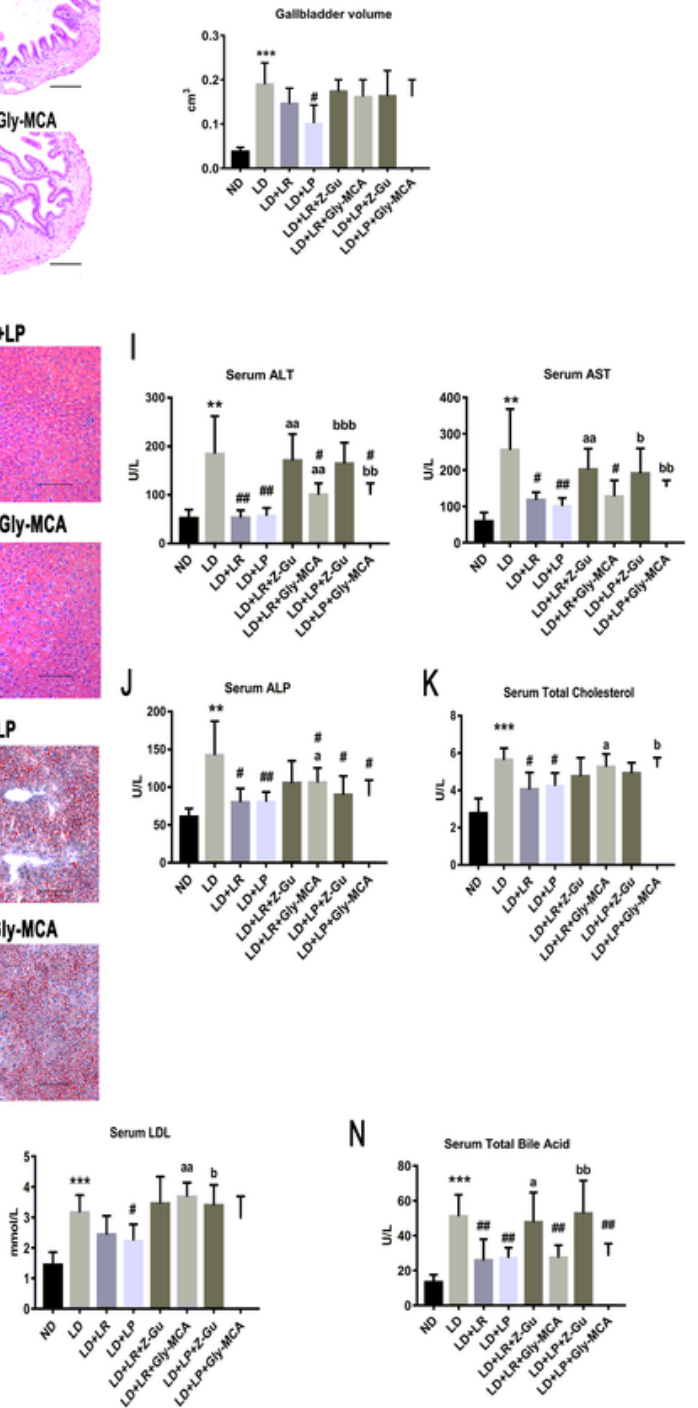

N

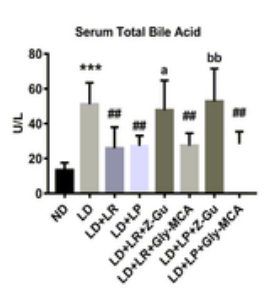

\section{Figure 5}

Inhibition of FXR activation attenuated the protective effects of LR and LP in LD-fed mice. Six mice were randomly assigned to each group fed a ND or an LD with or without LR or LP treatment (109 CFU/day) for 8 weeks. At the beginning of the LR or LP treatment, the mice were gavaged with (Z)-guggulsterone (ZGu), a global FXR inhibitor, or glycine- $\beta$-muricholic acid (Gly-MCA), an intestine-specific FXR inhibitor for 8 weeks. (A) Gross appearance of gallbladders and gallstones of mice administered different treatments. 
(B) Percentage of gallstone incidence in each group of mice. (C) The grade of experimental CGS in mice was based on visualized cholelithiasis. (D) Body weight of mice was recorded on the last week. (E) The ratio of liver weight to body weight. (F) Representative images of H\&E-stained gallbladder sections (×200). (G) Gallbladder volume estimated by the length, diameter and circumference of the gallbladders. (H) Representative images of H\&E-stained and Oil red O-stained liver sections ( $\times 200)$. (I-N) Serum ALT AST, ALP TC, TG, HDL, LDL and total bile acid were determined by a SIEMENS fast automatic biochemical analyzer (ADVIA 2400). Data are presented as the mean \pm SEM from at least three independent experiments $(n=6), 0.01<P \leq 0.05$, ** $0.001<P \leq 0.01$, *ᄎ* $P \leq 0.001$ vs ND, $\# 0.01<P \leq$ 0.05 , \#\# $0.001<P \leq 0.01$, \#\#\# $\mathrm{P} \leq 0.001$ vs LD.

\section{Supplementary Files}

This is a list of supplementary files associated with this preprint. Click to download.

- SFig1.tif

- SFig2.tif

- SFig3.tif

- SFig4.tif

- SFig5.tif

- SupplementaryTable1PCRGenesPrimersSequences.docx

- Supplementary.docx 